MATHEMATICAL MODELLING OF

POPULATION DYNAMICS

BANACH CENTER PUBLICATIONS, VOLUME 63

INSTITUTE OF MATHEMATICS

POLISH ACADEMY OF SCIENCES

WARSZAWA 2004

\title{
SEMILINEAR PERTURBATIONS OF HILLE-YOSIDA OPERATORS
}

\author{
HORST R. THIEME and HAUKE VOSSELER \\ Department of Mathematics and Statistics, Arizona State University \\ Tempe, AZ 85287-1804, U.S.A. \\ E-mail: h.thieme@asu.edu
}

\begin{abstract}
The semilinear Cauchy problem

$$
u^{\prime}(t)=A u(t)+G(u(t)), \quad u(0)=x \in \overline{D(A)},
$$
\end{abstract}

with a Hille-Yosida operator $A$ and a nonlinear operator $G: D(A) \rightarrow X$ is considered under the assumption that

$$
\|G(x)-G(y)\| \leq\|B(x-y)\| \quad \forall x, y \in D(A)
$$

with some linear $B: D(A) \rightarrow X$,

$$
B(\lambda-A)^{-1} x=\lambda \int_{0}^{\infty} e^{-\lambda t} V(s) x d s,
$$

where $V$ is of suitable small strong variation on some interval $[0, \varepsilon)$. We will prove the existence of a semiflow on $[0, \infty) \times \overline{D(A)}$ that provides Friedrichs solutions in $L_{1}$ for (1). If $X$ is a Banach lattice, we replace the condition above by

$$
|G(x)-G(y)| \leq B v \quad \text { whenever } x, y, v \in D(A),|x-y| \leq v,
$$

with $B$ being positive. We illustrate our results by applications to age-structured population models.

1. Introduction. Let $A$ be a Hille-Yosida operator in a Banach space $X$, i.e., after equivalent renormalization and an identity shift, an $m$-dissipative linear operator. Equivalently it is the generator of a locally Lipschitz continuous integrated semigroup $S$. The part $A_{0}$ of $A$ in $\overline{D(A)}$ generates a $C_{0}$-semigroup $\dot{S}$ which is the strong derivative of $S$.

We will consider perturbations $A+G$ with nonlinear operators $G$ that are Lipschitz continuous with respect to $A$ in various ways which we will make precise later, and show that the solutions of the associated Cauchy problem induce a continuous semiflow

2000 Mathematics Subject Classification: Primary 47J35; Secondary 92D25.

Research of the first author partially supported by NSF grant DMS-9706787.

Research of the second author supported by Studienstiftung des deutschen Volkes.

The paper is in final form and no version of it will be published elsewhere. 
(nonlinear semigroup) on $\overline{D(A)}$. This will generalize results that have been obtained for the following three scenarios:

- $G$ satisfies a Lipschitz condition on $\overline{D(A)}[30,11,21]$,

- $A$ is densely defined and $G$ a linear operator of Miyadera-Voigt type [22, 35],

- $X$ is an abstract L space and $G$ a positive linear operator on $D(A)[36,32]$.

Our approach does not cover the case that $A$ is $m$-dissipative, $D(A)$ is dense, $G$ is continuous, $D(G)=X$, and $G$ continuous and dissipative [38].

For the sake of exposition let us assume that $A$ is the generator of a $C_{0}$ semigroup $T_{0}$. The nonlinear nature of the perturbation does not allow the use of Dyson-Phillips series like in $[35]$ in constructing the semiflow $\Theta$,

$$
\Theta(t, x)=T_{0}(t) x+\int_{0}^{t} T_{0}(s) G(\Theta(t-s, x)) d s .
$$

Banach's fixed point theorem will be the basic tool of this paper, but it will not be applied to the previous integral equation, but to

$$
v(t)=G\left(T_{0}(t) x+\int_{0}^{t} T_{0}(s) v(t-s) d s\right)
$$

and $\Theta$ is obtained via

$$
\Theta(t, x)=T_{0}(t) x+\int_{0}^{t} T_{0}(s) v(t-s) d s .
$$

Actually we do not solve (2), but something more complicated. For $x \in \overline{D(A)}, u(t)=$ $\Theta(t, x)$ is the solution of the Cauchy problem

$$
u^{\prime}=A u+G \circ u, \quad t>0, \quad u(0)=x
$$

in a generalized integral sense which we will explain later (Section 2) and in an $L_{1^{-}}$ Friedrichs sense:

$\left(\mathbf{L}_{1}-\mathbf{F}\right) \quad u:[0, \infty) \rightarrow \overline{D(A)}$ is continuous, $u(0)=x$, and there exists a sequence of continuously differentiable functions $\left(u_{j}\right)$ with values in $D(A)$ such that $A u_{j}$ is continuous and

$$
\left.\begin{array}{c}
u_{j} \rightarrow u, \quad j \rightarrow \infty, \text { uniformly on }[0, T] \\
\left(u_{j}^{\prime}-A u_{j}\right) \text { and }\left(G \circ u_{j}\right) \text { converge in } L_{1}([0, T], X) \\
\lim _{j \rightarrow \infty}\left(u_{j}^{\prime}-A u_{j}\right)=\lim _{j \rightarrow \infty} G \circ u_{j}
\end{array}\right\} \quad \forall T>0 .
$$

We are going to carry out this program in detail in two situations. In either scenario, we will consider nonlinearities that are Lipschitz continuous relatively to $A$ in a global sense. While this can be an unpleasant restriction in some applications, it makes the semiflow satisfy an exponential Lipschitz condition in the state variable:

$$
\|\Theta(t, x)-\Theta(t, \tilde{x})\| \leq N e^{\theta t}\|x-\tilde{x}\| \quad \forall t \geq 0, x, \tilde{x} \in \overline{D(A)},
$$

with constants $\theta \in \mathbf{R}, N \geq 1$.

First, in the general case that $X$ is a Banach space (Section 5), we consider a nonlinear operator $G: D(A) \rightarrow X$ such that

$$
\|G(x)-G(y)\| \leq\|B(x-y)\| \quad \forall x, y \in D(A)
$$


for some linear operator $B: D(A) \rightarrow Y$ into a Banach space $Y$ such that

$$
B \int_{0}^{t} T_{0}(s) x d s=V(t) x, \quad x \in X,
$$

where $V$ is an operator family of suitable small strong variation on some interval $[0, \varepsilon]$.

As a special case we obtain the following nonlinear Miyadera-Voigt type result (see Section 6 for a more general and detailed result).

Theorem 1. Let $A$ be the generator of a $C_{0}$-semigroup $T_{0}$ on a Banach space $X$. Consider a Banach space $Y$, a dense subspace $D$ of $X$ and maps $G_{0}: D \rightarrow X, B_{0}: D \rightarrow Y$, $B_{0}$ linear, such that

$$
\left\|G_{0}(x)-G_{0}(y)\right\| \leq\left\|B_{0}(x-y)\right\| \quad \forall x, y \in D,
$$

and the following conditions are satisfied:

1. $D \subseteq D(A), \quad T_{0}(t) D \subseteq D$, and the function $B_{0} T_{0}(\cdot) x$ is continuous for all $x \in D$.

2. There are $\alpha, \lambda>0$ and $\gamma \in(0,1)$ such that

$$
\int_{0}^{\alpha} e^{-\lambda t}\left\|B_{0} T_{0}(t) x\right\| d t \leq \gamma\|x\| \quad \forall x \in D .
$$

Then there is a unique $A$-continuous extension $G: D(A) \rightarrow X$ of $G_{0}$ and a unique continuous semiflow $\Theta$ on $X$ which satisfies the exponential Lipschitz condition (5) such that, for each $x \in X, u(t)=\Theta(t, x)$ uniquely solves the Cauchy-problem (3) in the Friedrichs sense $\left(L_{1}-F\right)$.

In a second scenario, we concentrate on the Banach lattice case and a resolvent positive Hille-Yosida operator $A$. As illustration we present a result for the case that $X$ is an abstract $L$ space. For a general Banach lattice our assumptions become more involved (Section 7).

THEOREM 2. Let $A$ be a resolvent positive Hille-Yosida operator in the abstract L-space $X$. Consider a nonlinear $G: D(A) \rightarrow X$ and a linear positive $B: D(A) \rightarrow X$ such that

$$
|G(x)-G(y)| \leq B v \quad \text { whenever } x, y, v \in D(A),|x-y| \leq v,
$$

and the spectral radius of $B(\lambda-A)^{-1}$ is smaller than 1 for some $\lambda>s(A)$.

Then there is a unique continuous semiflow $\Theta$ on $\overline{D(A)}$ which satisfies the exponential Lipschitz condition (5) such that, for each $x \in \overline{D(A)}, u(t)=\Theta(t, x)$ uniquely solves the Cauchy-problem (3) in the Friedrichs sense $\left(L_{1}-F\right)$.

Moreover, if $G$ is positive, so is $\Theta$; more precisely: If $G$ maps $D(A) \cap X_{+}$into $X_{+}$, then $\Theta(t, \cdot)$ maps $\overline{D(A)} \cap X_{+}$into itself.

The exponential estimate (5) is reminiscent of the Crandall-Liggett theorem [9] and its extensions (see [6] and the references there) such that one might wonder whether Theorem 1 and 2 could be obtained therefrom. However, even for linear $G$, it has not been possible to show directly that $A+G$ is a Hille-Yosida operator, and the respective results were obtained by constructing the (integrated) semigroup [35, 32].

We will not be able to write the nonlinear integral equation (2) in this strict form for the simple reason that, in general, the expression in $(\cdot)$ is not an element of $D(A)$. We 
will rather work with fixed points of the following operator.

$$
\begin{aligned}
& \Psi_{x}(v)(t)=\lim _{h \rightarrow 0} G\left(\frac{1}{h}[S(t+h) x-S(t) x]+\frac{1}{h}[(S * v)(t+h)-(S * v)(t)]\right), \\
& (S * v)(t)=\int_{0}^{t} S(r) v(t-r) d r
\end{aligned}
$$

where the convergence is required to hold in the $L_{1}$-sense and $S$ is the integrated semigroup generated by $A$. In Section 2, we give abstract conditions under which the operator $\Psi_{x}$ is well defined and has fixed points which we relate to Friedrichs solutions and integral solutions of the semilinear Cauchy problem (3). They will be connected to the results presented above by Stieltjes convolutions (Section 3) and cumulative outputs (Section 4).

Once it is known that the generalized solutions of the Cauchy problem induce a semiflow, the powerful theory of dynamical system is available to study their qualitative behavior ([15, 27], e.g.).

In Section 8, we apply our results to age-structured population models where the births rate does not continuously depend on the population age density.

While, for the ease of exposition, we have restricted this paper to time-autonomous perturbations $A+G$, generalizations to non-autonomous semilinear perturbations $A+G(t)$ seem to be straightforward. Of course, the semiflow in (5) will be non-autonomous as well, a nonlinear evolutionary system, and a suitable generalization of the exponential Lipschitz condition will only hold for all times if the Lipschitz conditions for $G(t)$ will be uniform for all $t \geq 0$.

2. Nonlinear perturbations in Banach spaces. Let $X$ be a Banach space and $A$ a Hille-Yosida operator in $X$, i.e., $A$ is a linear closed operator such that the resolvent set $\rho(A)$ of $A$ contains an infinite interval $(\omega, \infty)$ and there exists some $M \geq 1$ such that

$$
\left\|(\lambda-A)^{-n}\right\| \leq M(\lambda-\omega)^{-n} \quad \forall \lambda>\omega, n \in \mathbf{N} .
$$

Equivalently $[2,17,1], A$ generates an integrated semigroup $S(t), t \geq 0$, such that

$$
\|S(t)-S(r)\| \leq M \int_{r}^{t} e^{\omega s} d s .
$$

In general, an integrated semigroup is a strongly continuous family $S$ of bounded linear operators $S(t), t \geq 0$, that satisfies $S(0)=0$ and the functional equation

$$
S(t) S(r)=\int_{0}^{r+t} S(s) d s-\int_{0}^{t} S(s) d s-\int_{0}^{r} S(s) d s, \quad t, r \geq 0,
$$

strongly. The fact that $A$ generates $S$ can be either expressed as

$$
x \in D(A), y=A x \Leftrightarrow S(t) x-t x=\int_{0}^{t} S(r) y d r \forall t \geq 0,
$$

[29] or, if $S$ is exponentially bounded, equivalently in terms of Laplace transforms as

$$
(\lambda-A)^{-1}=\lambda \int_{0}^{\infty} e^{-\lambda t} S(t) d t, \quad \lambda>\omega,
$$

$[2,17,23,1]$. If $A$ is a Hille-Yosida operator, then $S(t) x$ is strongly differentiable in $t \geq 0$ if and only if $x \in \overline{D(A)}$. In this case, $\dot{S}(t) x:=\frac{d}{d t} S(t) x$ is a $C_{0}$-semigroup on $\overline{D(A)}$ which 
is generated by the part of $A$ in $\overline{D(A)}$. The following result in [17] is one of the linear foundations for our nonlinear considerations.

Theorem 3. Let $f \in L_{1}([0, T], X)$. Define

$$
(S * f)(t)=\int_{0}^{t} S(r) f(t-r) d r, \quad t \geq 0 .
$$

Then $S * f$ is continuously differentiable, takes its values in $D(A)$ with $A(S * f)(t)$ being a continuous function in $t \geq 0$, and $u=(S * f)^{\prime}$ is the unique solution of

$$
u(t)=A \int_{0}^{t} u(s) d s+\int_{0}^{t} f(s) d s, \quad t \in[0, T] .
$$

Finally

$$
\left\|(S * f)^{\prime}(t)\right\| \leq M \int_{0}^{t} e^{\omega r}\|f(t-r)\| d r \quad \forall t \in[0, T] .
$$

The result that (9) has a unique solution which satisfies the estimate in Theorem 3 has already been obtained in [10].

In the following we will write

$$
S \diamond f:=(S * f)^{\prime} .
$$

After this linear prelude, let $Z$ be a closed subspace of $X$ and

$$
G: D(A) \rightarrow Z
$$

be $A$-continuous, i.e., if $X_{A}$ denotes $D(A)$ equipped with the graph norm, $\|x\|_{A}=\|x\|+$ $\|A x\|$, then $G: X_{A} \rightarrow Z$ is continuous. We consider the semilinear Cauchy problem

$$
u^{\prime}(t)=(A+G) u(t), \quad u(0)=x \in \overline{D(A)} .
$$

We will use two closely connected concepts of solving (10) in a generalized sense. Up to slight modifications, they can be found in [10] and [5].

Definition 1. Let $T>0$. A continuous function $u:[0, T] \rightarrow X$ with $u(0)=x$ is called a Friedrichs solution or an F-solution in $L_{1}$ of (10) on $[0, T]$ if there are functions $u_{j} \in C^{1}([0, T], X) \cap C\left([0, T], X_{A}\right)$ such that $\left(G u_{j}\right)_{j \in \mathbf{N}}$ converges in $L_{1}([0, T], X)$ and

$$
\begin{aligned}
& u_{j} \rightarrow u, \quad j \rightarrow \infty, \text { uniformly on }[0, T], \\
& \left(u_{j}^{\prime}-A u_{j}\right) \text { and }\left(G \circ u_{j}\right) \text { converge in } L_{1}([0, T], X), \\
& \lim _{j \rightarrow \infty}\left(u_{j}^{\prime}-A u_{j}\right)=\lim _{j \rightarrow \infty} G \circ u_{j} .
\end{aligned}
$$

The second notion is a modification of the concept of integral solution.

Definition 2. A continuous function $u:[0, T] \rightarrow X$ is called an I-solution of (10) if $u$ can be continuously extended to some interval $[0, T+\epsilon], \epsilon>0$, such that $\int_{0}^{t} u(s) d s \in D(A)$ for all $t \in[0, T+\epsilon], G\left(\frac{1}{h} \int_{t}^{t+h} u(s) d s\right)$ converges in $L_{1}([0, T] ; X)$ as $h \rightarrow 0$, and

$$
u(t)=x+A \int_{0}^{t} u(s) d s+\lim _{h \rightarrow 0} \int_{0}^{t} G\left(\frac{1}{h} \int_{s}^{s+h} u(r) d r\right) d s \quad \forall t \in[0, T] .
$$

We establish a first relation between the two solution concepts.

LEMma 4. If $0<T_{1}<T_{2}$, then any $I$-solution on $\left[0, T_{2}\right]$ is an $F$-solution in $L_{1}$ on $\left[0, T_{1}\right]$. 
Proof. Let $u$ be an $I$-solution on $\left[0, T_{2}\right]$. Set

$$
v(t)=\lim _{h \rightarrow \infty} G\left(\frac{1}{h} \int_{t}^{t+h} u(s) d s\right), \quad t \in\left[0, T_{2}\right],
$$

with convergence holding in $L_{1}\left(\left[0, T_{2}\right] ; Z\right)$. Then

$$
u(t)=x+A \int_{0}^{t} u(s) d s+\int_{0}^{t} v(s) d s \quad \forall t \in\left[0, T_{2}\right] .
$$

Define

$$
u_{j}(t)=j \int_{t}^{t+\frac{1}{j}} u(s) d s, \quad v_{j}(t)=j \int_{t}^{t+\frac{1}{j}} v(s) d s, \quad t \in\left[0, T_{1}\right] .
$$

Then $u_{j} \rightarrow u$ in $C\left(\left[0, T_{1}\right] ; X\right)$ and $v_{j} \rightarrow v$ in $L_{1}\left(\left[0, T_{1}\right] ; Z\right)$. Further $v=\lim _{j \rightarrow \infty} G \circ u_{j}$ in $L_{1}\left(\left[0, T_{1}\right] ; X\right)$. Finally

$$
u_{j}^{\prime}(t)=j\left(u\left(t+\frac{1}{j}\right)-u(t)\right)=A u_{j}(t)+v_{j}(t),
$$

SO

$$
u_{j}^{\prime}(t)-A u_{j}(t)=v_{j}(t) \rightarrow v(t) \leftarrow G\left(u_{j}(t)\right)
$$

in $L_{1}\left(\left[0, T_{1}\right] ; Z\right)$.

Theorem 3 suggests looking for $I$-solutions of (10) as solutions of the integral equation

$$
u(t)=\dot{S}(t) x+\lim _{h \rightarrow 0} \frac{d}{d t} \int_{0}^{t} S(s) G\left(\frac{1}{h} \int_{t-s}^{t-s+h} u(r) d r\right) d s,
$$

where $S$ is the integrated semigroup generated by $A$. Therefore the following mapping $\Psi$ from $\overline{D(A)} \times L_{1}([0, T], Z)$ to $L_{1}([0, T], Z)$ will play an important role whenever it is well-defined,

$$
\Psi(x, v)(t)=\lim _{h \rightarrow 0} G\left(\frac{1}{h} \int_{t}^{t+h} \dot{S}(s) x d s+\frac{1}{h} \int_{t}^{t+h}(S \diamond v)(s) d s\right) .
$$

The convergence is required to be in $L_{1}([0, T], Z)$ and to be independent of how $v$ is extended outside of $[0, T]$. Notice that this is the same map as in (6). We have the following fundamental result.

Proposition 5. Assume the operator $\Psi$ in (12) is well-defined and let $T>0, x \in$ $\overline{D(A)}, \Psi_{x}=\Psi(x, \cdot)$. Then there is a one-to-one correspondence between fixed points of $\Psi_{x}$ and I-solutions. More precisely, the following two relations hold:

(1) If $v_{x}$ is a fixed point of $\Psi_{x}$ in $L_{1}([0, T], Z)$ and is extended by 0 outside of $[0, T]$, then $u=\dot{S}(\cdot) x+S \diamond v_{x}$ is an I-solution on $[0, T]$.

(2) If $u$ is an I-solution of $[0, T]$, then

$$
v(t)=L_{1}-\lim _{h \rightarrow 0} G\left(\frac{1}{h} \int_{t}^{t+h} u(s) d s\right)
$$

is a fixed point of $\Psi_{x}$ in $L_{1}([0, T], Z)$ and $u=\dot{S}(\cdot) x+S \diamond v$. 
Proof. (1) Let $v_{x}$ be a fixed point of $\Psi_{x}$ and set $u=\dot{S}(\cdot) x+S \diamond v_{x}$. Extend $v_{x}$ by 0 outside of $[0, T]$. By definition of $\Psi, v_{x}(t)=\lim _{h \rightarrow 0} G\left(\frac{1}{h} \int_{t}^{t+h} u(r) d r\right)$. $u$ is an $I$-solution by Theorem 3 .

(2) Let $u$ be an $I$-solution of (10), i.e., satisfy (11) or rather all of Definition 2. Define $v$ as stated in the theorem, then $u(t)=\dot{S}(t) x+(S \diamond v)(t)$ for $t \in[0, T]$ by Theorem 3 . Since, by assumption, $\Psi$ is well-defined and independent of how $v$ is extended, $v$ is a fixed point of $\Psi_{x}$.

Proposition 5 suggests looking for conditions which make the operator $\Psi$ given by (12) well defined. The following subspace $E$ of $\overline{D(A)} \times L_{1}([0, T] ; Z)$ will play an important role.

By definition, $E$ consists of those pairs $(x, v)$ such that

$$
\left\{\begin{array}{l}
x \in \overline{D(A)}, \quad v \in L_{1}([0, T], Z), \\
\dot{S}(t) x+(S \diamond v)(t) \in D(A) \quad \forall t \in[0, T] \\
A(\dot{S}(t) x+(S \diamond v)(t)) \text { is a continuous function of } t \in[0, T] .
\end{array}\right.
$$

Since $G: D(A) \rightarrow Z$ is $A$-continuous,

$$
\Psi_{0}(x, v)(t)=G(\dot{S}(t) x+(S \diamond v)(t))
$$

defines a map $\Psi_{0}$ from $E$ to $C([0, T] ; Z) \subseteq L_{1}([0, T] ; Z)$. Notice that the argument of $G$ is in $D(A)$ and is a continuous function of $t$ in the graph norm of $A$.

Proposition 6. Let $G: D(A) \rightarrow Z$ be A-continuous and $E$ be the subspace defined in (13). Assume that $\Psi_{0}$ can be extended to a continuous map $\Psi$ from $\overline{D(A)} \times L_{1}([0, T] ; Z)$ to $L_{1}([0, T] ; Z)$. Then $\Psi$ satisfies (12).

Proof. Set

$$
w_{h}(t)=\frac{1}{h}(S(t+h)-S(t)) x+\frac{1}{h} \int_{t}^{t+h}(S \diamond v)(s) d s .
$$

Here we have extended $v$ beyond $T$ in an arbitrary way such that $v \in L_{1}([0, T+\epsilon], X)$ for some $\epsilon>0$. By Theorem 3, $w_{h}(t) \in D(A)$, and

$$
A w_{h}(t)=\frac{1}{h}(\dot{S}(t+h)-\dot{S}(t)) x+\frac{1}{h}((S \diamond v)(t+h)-(S \diamond v)(t))-\frac{1}{h} \int_{t}^{t+h} v(s) d s
$$

is a continuous function of $t \in[0, T]$. Moreover

$$
\begin{aligned}
w_{h}(t)= & \frac{1}{h} \dot{S}(t) S(h) x+\frac{1}{h}((S * v)(t+h)-(S * v)(t)) \\
= & \frac{1}{h} \dot{S}(t) S(h) x+\frac{1}{h} \int_{0}^{t} S(s)(v(t+h-s)-v(t-s)) d s \\
& +\frac{1}{h} \int_{t}^{t+h} S(s) v(t+h-s) d s \\
= & \frac{1}{h} \dot{S}(t) S(h) x+\frac{1}{h} \int_{0}^{t} S(s) \frac{d}{d t}\left(\int_{t-s}^{t+h-s} v(r) d r\right) d s \\
& +\frac{1}{h} \int_{0}^{h} S(t+s) v(h-s) d s
\end{aligned}
$$




$$
\begin{aligned}
= & \frac{1}{h} \dot{S}(t) S(h) x+\frac{d}{d t} \int_{0}^{t} S(s)\left(\frac{1}{h} \int_{t-s}^{t+h-s} v(r) d r\right) d s \\
& +\frac{1}{h} \int_{0}^{h}(S(t+s)-S(t)) v(h-s) d s \\
= & \dot{S}(t) x_{h}+\left(S \diamond v_{h}\right)(t)
\end{aligned}
$$

with

$$
x_{h}=\frac{1}{h} S(h) x+\frac{1}{h} \int_{0}^{h} S(s) v(h-s) d s, \quad v_{h}(t)=\frac{1}{h} \int_{t}^{t+h} v(r) d r .
$$

Since $w_{h}(t) \in D(A)$ and $A w_{h}(t)$ is continuous in $t$, by (13),

$$
\left(x_{h}, v_{h}\right) \in E \quad \text { and } \quad G\left(w_{h}(t)\right)=\Psi_{0}\left(x_{h}, v_{h}\right)(t) .
$$

As $x_{h} \rightarrow x$ and $v_{h} \rightarrow v$ in $L_{1}([0, T], Z)$ as $h \rightarrow 0$ and $\Psi$ is the continuous extension of $\Psi_{0}$ by assumption, we have

$$
\Psi(x, v)=\lim _{h \rightarrow 0} G \circ w_{h} \quad \text { in } L_{1}([0, T] ; Z) .
$$

Proposition 7. Suppose that $T>0$ and the assumptions of Proposition 6 hold. If $u$ is an F-solution of (9) on $[0, T]$, then $u=\dot{S}(\cdot) x+S \diamond v$ with a fixed point $v$ of $\Psi_{x}$ and $u$ is an I-solution of (9).

Proof. Let $u$ be an $F$-solution in $L_{1}$. Then we find $u_{j} \in C^{1}([0, T], X) \cap C\left([0, T], X_{A}\right)$, $w_{j} \in L_{1}([0, T], X)$ such that $\left(G u_{j}\right)_{j \in \mathbf{N}}$ converges in $L_{1}([0, T], X)$ and

$$
\begin{aligned}
& \lim _{j \rightarrow \infty}\left\|u_{j}-u\right\|=0, \\
& \lim _{j \rightarrow \infty}\left\|w_{j}\right\|_{1}=0
\end{aligned}
$$

and

$$
u_{j}^{\prime}=A u_{j}+G \circ u_{j}+w_{j} .
$$

We set $v_{j}:=G \circ u_{j}$. By Theorem 3,

$$
u_{j}=\dot{S}(\cdot) u_{j}(0)+S \diamond\left(G \circ u_{j}+w_{j}\right)=\dot{S}(\cdot) u_{j}(0)+S \diamond v_{j}+S \diamond w_{j} .
$$

Since $u_{j} \in C\left([0, T], X_{A}\right),\left(u_{j}(0), v_{j}+w_{j}\right)$ is an element of $E$ by (13), and we have the following representation of $v_{j}$,

$$
v_{j}=G \circ\left(\dot{S}(\cdot) u_{j}(0)+S \diamond v_{j}+S \diamond w_{j}\right)=\Psi_{0}\left(u_{j}(0), v_{j}+w_{j}\right) .
$$

Since $\Psi$ is continuous and $u_{j}(0) \rightarrow u(0),\left(v_{j}+w_{j}\right) \rightarrow v$ in $L_{1}$, we have

$$
\Psi(u(0), v)=\lim _{j \rightarrow \infty} \Psi_{0}\left(u_{j}(0), v_{j}+w_{j}\right)=\lim _{j \rightarrow \infty} v_{j}=v
$$

in $L_{1}([0, T], X)$ and $v$ is a fixed point of $\Psi_{x}, x=u(0)$. Furthermore, by (15),

$$
\begin{aligned}
u(t) & =\lim _{j \rightarrow \infty} u_{j}(t)=\lim _{j \rightarrow \infty} \dot{S}(\cdot) u_{j}(0)+\left(S \diamond v_{j}\right)(t)+\left(S \diamond w_{j}\right)(t) \\
& =\dot{S}(\cdot) u(0)+S \diamond v .
\end{aligned}
$$

Then $u$ is an $I$-solution by Proposition 5, Part 1 . 
Once we have found the unique fixed point $v_{x}$ of $\Psi_{x}$ in $L_{1}([0, T], Z)$, motivated by Propositions 6 and 7, we define

$$
\Theta(t, x)=\dot{S}(t) x+\left(S \diamond v_{x}\right)(t) .
$$

TheOREM 8. Let $T>0$ and assume that $L_{1}([0, T], Z)$ can equivalently be renormalized such that

$$
\left\|\Psi_{0}(x, v)-\Psi_{0}(y, w)\right\| \leq K_{1}\|x-y\|+K_{2}\|v-w\| \quad \forall(x, v),(y, w) \in E,
$$

where $K_{1}>0$ and $K_{2} \in(0,1)$.

Then there exists a continuous semiflow $\Theta$ on $[0, \infty) \times \overline{D(A)}$ such that the exponential Lipschitz condition (5) is satisfied and $u(t)=\Theta(t, x)$ are the unique I-solutions and the unique F-solutions of (10) on every finite interval $[0, \tau]$.

Proof. Since $E$ contains the dense subspace $D\left(A_{0}\right) \times C_{0}^{\infty}((0, T) ; Z)$ of the Banach space $\overline{D(A)} \times L_{1}([0, T] ; Z), \Psi_{0}$ has a Lipschitz extension $\Psi$ to the latter space with the same Lipschitz constants.

$\Psi$ is a uniform contraction on $\overline{D(A)} \times L_{1}([0, T] ; Z)$ and, by the parameter version of Banach's fixed point theorem, there are unique fixed points $v_{x}$ of the mappings $\Psi_{x}$ for all $x \in \overline{D(A)}$ and the dependence of the $v_{x}$ on $x$ is Lipschitz continuous on $[0, T]$ (see e.g. the proof of [8], Th. 2.2),

$$
\left\|v_{x}-v_{y}\right\|_{1} \leq C\|x-y\| \quad \forall x, y \in \overline{D(A)},
$$

with a suitable constant $C>0$. We define $\Theta$ as in (17). By Theorem 3 , for $0<t \leq T$,

$$
\begin{aligned}
\|\Theta(t, x)-\Theta(t, y)\| & \leq \sup _{s \in[0, T]}\|\dot{S}(s)\|\|x-y\|+\left\|S \diamond\left(v_{x}-v_{y}\right)\right\| \\
& \leq M e^{\omega_{+} T}\left(\|x-y\|+\left\|v_{x}-v_{y}\right\|_{1}\right) \leq K\|x-y\|,
\end{aligned}
$$

where $K=M e^{\omega_{+} T}(1+C)$ and $\omega_{+}=\max \{\omega, 0\}$ is the positive part of $\omega$. From Propositions 5 and 6 we know that $\Theta(\cdot, x)$ is the unique $I$-solution of $(10)$.

The semiflow property follows from the fact that $u=\Theta(\cdot, x)$ is also the unique $F$ solution of (10). See Lemma 4 and Lemma 7. Notice that $\tilde{u}(t)=\Theta(t+s, x)$ is the $F$ solution of (10) with initial value $\tilde{u}(0)=\Theta(s, x)$, so $\Theta(t+s, x)=\Theta(t, \Theta(s, x))$.

The semiflow $\Theta$ can be extended to $[0,2 T]$ in the standard way: Let $t=s+r \in(T, 2 T]$ with $r, s \in[0, T]$. Set $\Theta(t, x)=\Theta(r, \Theta(s, x))$. The semiflow property on $[0, T]$ guarantees that this definition is independent of the choice of $r$ and $s$ and readily extends to $[0,2 T]$. It also implies that $\Theta(\cdot, x)$ is an $I$-solution on $[0,2 T]$,

$$
\begin{aligned}
\Theta(T+t, x)-x= & \Theta(t, \Theta(T, x))-x \\
= & \Theta(T, x)-x+A \int_{0}^{t} \Theta(s, \Theta(T, x)) d s \\
& +\lim _{h \searrow 0} \int_{0}^{t} G\left(\frac{1}{h} \int_{s}^{s+h} \Theta(r, \Theta(T, x)) d r\right) d s
\end{aligned}
$$




$$
\begin{aligned}
= & A \int_{0}^{T} \Theta(s, x) d s+\lim _{h \searrow 0} \int_{0}^{T} G\left(\frac{1}{h} \int_{s}^{s+h} \Theta(r, x) d r\right) d s \\
& +A \int_{0}^{t} \Theta(s+T, x) d s+\lim _{h \searrow 0} \int_{0}^{t} G\left(\frac{1}{h} \int_{s}^{s+h} \Theta(r+T, x) d r\right) d s \\
= & A \int_{0}^{T+t} \Theta(s, x) d s+\lim _{h \searrow 0} \int_{0}^{T+t} G\left(\frac{1}{h} \int_{s}^{s+h} \Theta(r, x) d r\right) d s,
\end{aligned}
$$

and the limit exists in $L_{1}$. The Lipschitz continuity of $\Theta(t, x)$ in $x$ easily extends to [T, 2T] with the Lipschitz constant $K^{2}$. Continuing this way we can extend the semiflow to $[0, \infty)$ with

$$
\|\Theta(t, x)-\Theta(t, y)\| \leq K^{n}\|x-y\| \quad \forall t \in[(n-1) T, n T), n \in \mathbf{N} .
$$

The exponential Lipschitz condition (5) is satisfied with $N=K, \theta=\frac{\ln K}{T}$.

Corollary 9. Let $T>0$ and assume that $L_{1}([0, T], Z)$ can equivalently be renormalized such that

$$
\left\|\Psi_{0}(x, v)-\Psi_{0}(y, w)\right\| \leq K_{1}\|x-y\|+K_{2}\|v-w\| \quad \forall(x, v),(y, w) \in E,
$$

where $K_{1}, K_{2}>0$. Then $\Psi_{0}$ has an extension $\Psi$ satisfying the analogous inequality on $\overline{D(A)} \times L_{1}([0, T], X)$.

Further assume that there exist $n \in \mathbf{N}$ and $\varepsilon \in(0,1)$ such that for $\Psi_{x}=\Psi(x, \cdot)$,

$$
\left\|\Psi_{x}^{n}(v)-\Psi_{x}^{n}(w)\right\| \leq \varepsilon\|v-w\| \quad \forall v, w \in L_{1}([0, T], Z), x \in \overline{D(A)} .
$$

Then there is a unique continuous semiflow $\Theta$ on $[0, \infty) \times \overline{D(A)}$ such that the exponential Lipschitz condition (5) is satisfied and $u(t)=\Theta(t, x)$ are the unique I-solutions and the unique F-solutions of (10) on every finite interval $[0, \tau]$.

Proof. By assumption, with $\Psi_{x}:=\Psi(x, \cdot), \Psi_{x}^{n}$ is a strict contraction on the Banach space $L_{1}([0, T], Z)$ and so has a unique fixed point $v_{x}$. Since $\Psi_{x}\left(v_{x}\right)$ is also a fixed point of $\Psi_{x}^{n}$, $\Psi_{x}\left(v_{x}\right)=v_{x}$. Since every fixed point of $\Psi_{x}$ is also a fixed point of $\Psi_{x}^{n}, v_{x}$ is the unique fixed point of $\Psi_{x}$. For all $x, y \in \overline{D(A)}$,

$$
\begin{aligned}
\left\|v_{x}-v_{y}\right\| & =\left\|\Psi_{x}^{n}\left(v_{x}\right)-\Psi_{y}^{n}\left(v_{y}\right)\right\| \\
& \leq\left\|\Psi_{x}^{n}\left(v_{x}\right)-\Psi_{x}^{n}\left(v_{y}\right)\right\|+\left\|\Psi_{x}^{n}\left(v_{y}\right)-\Psi_{y}^{n}\left(v_{y}\right)\right\| \\
& \leq\left\|v_{x}-v_{y}\right\| \varepsilon+K_{n}\|x-y\|,
\end{aligned}
$$

for some constant $K_{n}>0$. Reorganizing this inequality,

$$
\left\|v_{x}-v_{y}\right\| \leq(1-\varepsilon)^{-1} K_{n}\|x-y\| .
$$

The remaining part of the corollary follows as in Theorem 8.

For cases in which the estimate in Theorem 8 is difficult to check directly, we offer the following version which will be used in the Banach lattice case. Let $\phi: \mathbf{R} \rightarrow[0, \infty)$ be a continuous non-negative function with compact support and $\int_{\mathbf{R}} \phi(s) d s=1$. Set

$$
\phi_{j}(t)=j \phi(j t),
$$

and for $v \in L_{1}([0, T) ; X)$ define

$$
v_{j}^{\phi}(t)=\int_{0}^{\infty} \phi_{j}(s-t) v(s) d s,
$$


where $v$ has been extended outside of $[0, T]$ in an arbitrary measurable essentially bounded fashion. One readily checks that $v_{j}^{\phi} \rightarrow v, j \rightarrow \infty$, uniformly on compact subsets of $(0, T)$, if $v$ is continuous on $(0, T)$, and $v_{j}^{\phi} \rightarrow v$ in $L_{1}([0, T] ; X)$. If $v:[0, T] \rightarrow D(A)$ and $A v$ is continuous on $(0, T)$ then $v_{j}^{\phi}(t) \in D(A)$ and $A v_{j}^{\phi} \rightarrow A v, j \rightarrow \infty$, uniformly on compact subsets of $(0, T) . \phi$ with the properties above is called a mollifier because $v^{\phi}$ is typically smoother than $v$.

Corollary 10. Let $G: D(A) \rightarrow Z$ be A-continuous and $E$ be the subspace defined in (13). Let $K_{1}, K_{2} \geq 0$ and $\phi$ a mollifier as above such that, for all $(x, v),(y, w) \in E$,

$$
\begin{aligned}
\liminf _{j \rightarrow \infty} \int_{0}^{T}\left\|G\left((\dot{S}(\cdot) x+S \diamond v)_{j}^{\phi}(t)\right)-G\left((\dot{S}(\cdot) y+S \diamond w)_{j}^{\phi}(t)\right)\right\| d t & \\
& \leq K_{1}\|x-y\|+K_{2} \int_{0}^{T}\|v(t)-w(t)\| d t .
\end{aligned}
$$

Then the map $\Psi_{0}$ in (14) satisfies the inequalities (18) in Corollary 9, and its extension $\Psi$ satisfies the analogous inequalities on $\overline{D(A)} \times L_{1}([0, T], X)$ and equation (12).

Proof. Since $G$ is $A$-continuous, we have for all $t \in(0, T)$ and $(x, v) \in E$

$$
\lim _{j \rightarrow \infty} G\left((\dot{S}(\cdot) x+S \diamond v)_{j}^{\phi}(t)\right)=G(\dot{S}(t) x+S \diamond v(t)) .
$$

By Fatou's lemma, the inequalities (18) in Corollary 9 holds, and $\Psi_{0}$ has an extension $\Psi$ on $\overline{D(A)} \times L_{1}([0, T], X)$ which satisfies the analogous inequalities and, by Proposition 6 , equation $(12)$.

\section{A Stieltjes type convolution of operator families with vector-valued $L_{1}$ -} functions. The convolution we introduce in this section turns out to be useful in the proof of our perturbation theorems. The results we present are established in [34].

Let $I \subseteq \mathbf{R}$ be an interval which is bounded from below and

$$
l(I):=\inf I \quad \text { and } \quad r(I):=\sup (I)
$$

denote its left and right end-point. Unless explicitly stated otherwise, we always assume that $l(I) \in I$ and $r(I) \notin I$. Recall that, for every $f \in L_{1}(I, X), \int_{l(I)}^{\cdot} f(t) d t$ is of bounded variation and its variation is given by

$$
\mathbf{v}\left(\int_{l(I)}^{\cdot} f(t) d t ; I\right)=\|f\|_{1}=\int_{l(I)}^{r(I)}\|f(s)\| d s .
$$

This motivates our next definition.

Definition 3. A function $f \in L_{1}(I, X)$ is of bounded pseudo-variation if

$$
\mathbf{p v}(f ; I):=\varlimsup_{h \searrow 0} \frac{1}{h} \int_{l(I)}^{r(I)-h}\|f(s+h)-f(s)\| d s<\infty .
$$

If $r(I)=\infty, r(I)-h$ is to be interpreted as $\infty$. Recall that $f: I \rightarrow X$ is of bounded variation if

$$
\mathbf{v}(f ; I):=\sup \left\{\sum_{j=1}^{k}\left\|f\left(t_{j}\right)-f\left(t_{j-1}\right)\right\|\right\}<\infty,
$$


where the supremum is taken over all partitions $t_{0}<\cdots<t_{k}$ of $I$ where $k \in \mathbf{N}$ and $t_{j} \in I . f: I \rightarrow X$ is Lipschitz continuous if

$$
\mathbf{p} \mathbf{v}_{\infty}(f ; I):=\sup \left\{\frac{\|f(t)-f(s)\|}{|t-s|} ; t, s \in I, t \neq s\right\}<\infty .
$$

Every function of bounded pseudo-variation admits a version that is of bounded variation:

Lemma 11. Let $f: I \rightarrow X$ be of bounded pseudo-variation. Then

$$
f^{\oplus}(t):=\lim _{h \searrow 0} \frac{1}{h} \int_{t}^{t+h} f(s) d s
$$

exists for all $t \in I, f=f^{\oplus}$ a.e., and $f^{\oplus}$ is of bounded variation on $I$ with $\mathbf{v}\left(f^{\oplus}, I\right)=$ $\mathbf{p v}(f, I)$. Further $\left(f^{\oplus}\right)^{\oplus}=f^{\oplus}$ and $f^{\oplus}$ is right continuous.

We set

$$
\mathbf{p v}^{\oplus}(f ; I):=\mathbf{p v}(f ; I)+\left\|f^{\oplus}(l(I))\right\| .
$$

Proposition 12. The vector space

$$
\mathcal{P V}(I ; X):=\left\{f^{\oplus} ; f: I \rightarrow X \text { is of bounded pseudo-variation }\right\}
$$

equipped with the norm $\mathbf{p v}^{\oplus}(f ; I)$ is a Banach space.

The analogous result for the space of Lipschitz continuous functions is well known. We extend the previous concepts to operator families.

Definition 4. A family of bounded linear operators $(U(t))_{t \in I}$ from a Banach space X into a Banach space $Y$ is called to be of bounded strong pseudo-variation if

$$
\mathbf{p v}(U(\cdot) x ; I)<\infty \quad \forall x \in X .
$$

It is easy to check that $x \mapsto U(\cdot) x$ is a closed linear operator from $X$ into the Banach space $\mathcal{P} \mathcal{V}(I ; Y)$ and so a bounded linear operator; this implies that

$$
\begin{aligned}
& \mathbf{P V}(U ; I, X):=\sup _{\|x\| \leq 1, x \in X} \mathbf{p v}(U(\cdot) x ; I)<\infty, \\
& \mathbf{P V}^{\oplus}(U ; I, X):=\sup _{\|x\| \leq 1, x \in X} \mathbf{p v}^{\oplus}(U(\cdot) x ; I)<\infty,
\end{aligned}
$$

and

$$
\begin{aligned}
& \mathbf{p v}(U(\cdot) x ; I) \leq \mathbf{P V}(U ; I, X)\|x\|, \\
& \mathbf{p v} \mathbf{v}^{\oplus}(U(\cdot) x ; I) \leq \mathbf{P} \mathbf{V}^{\oplus}(U ; I, X)\|x\|, \quad \forall x \in X .
\end{aligned}
$$

We call $\mathbf{P V}(U ; I, X)$ the strong pseudo-variation of $U$ on $I \times X$.

It is well known and follows in the same way that $U(\cdot)$ is Lipschitz continuous in operator norm if it is strongly Lipschitz continuous. We set

$$
\mathbf{P V}_{\infty}(U ; I, X)=\sup \left\{\frac{\|U(t)-U(t)\|}{|t-s|} ; t, s \in I, t \neq s\right\}
$$

We introduce

$$
U^{\oplus}(t) x=\lim _{h \downarrow 0} \frac{1}{h} \int_{t}^{t+h} U(s) x d s
$$


which exists according to Lemma 11 . Notice that $U(t) x$ and $U^{\oplus}(t) x$ have the same pseudovariation.

Before we define a Stieltjes type convolution of $U$ with $f \in L_{1}(I, X)$, with $l(I)=0$, we recall that the standard convolution

$$
(U * f)(t):=\int_{0}^{t} U(s) f(t-s) d s, \quad t \in I,
$$

defines a function in $L_{1}(I, Y)$, if $f \in L_{1}(I, X)$ and $U(t)$ is strongly integrable on $I$. If $U$ is of bounded strong pseudo-variation, we actually have the following result.

Proposition 13. Let I be a bounded interval, $U$ be of bounded strong pseudo-variation on $I$ and $f \in L_{1}([0, T], X)$. Then $U * f \in L_{\infty}(I, Y)$ and

$$
\|U * f\|_{\infty} \leq \sup _{\|x\| \leq 1}\|U(\cdot) x\|_{\infty}\|f\|_{1} .
$$

In fact, much more holds.

THEOREM 14. Let $U$ be of bounded strong pseudo-variation on $I$ and $f \in L_{1}(I, X)$. Then $U * f$ is continuous on I. Moreover the limit

$$
U \star f=\lim _{h \searrow 0} \frac{1}{h} \int_{0}^{\cdot}(U(s+h)-U(s)) f(\cdot-s) d s,
$$

exists in $L_{1}(I, Y)$,

$$
\|U \star f\|_{1} \leq \mathbf{P V}(U ; I, X)\|f\|_{1}
$$

and

$$
(U * f)(t)=\int_{0}^{t}(U \star f)(s) d s+\int_{0}^{t} U^{\oplus}(0) f(s) d s
$$

Finally

$$
U^{\oplus} \star f=U \star f
$$

We define

$$
(U \diamond f)(t)=(U \star f)(t)+U^{\oplus}(0) f(t) .
$$

Our Stieltjes type convolution relates to the standard convolution as follows.

Corollary 15. Let $I=[0, T)$ and $(U(t))_{t \in I}$ be a family of bounded linear operators from $X$ to $Y$ that is of bounded strong pseudo-variation.

(a) Then, for all $f \in L_{1}(I, Y)$,

$$
(U * f)(t)=\int_{0}^{t}(U \diamond f)(s) d s \quad \forall t \in I, \quad\|U \diamond f\|_{1} \leq \mathbf{P V}^{\oplus}(U ;[0, T), X)\|f\|_{1} .
$$

(b) If $f:[0, T) \rightarrow X$ is of bounded pseudo-variation, then $U \diamond f$ is of bounded pseudovariation on $[0, T)$,

$$
\mathbf{p v}^{\oplus}(U \diamond f ; I) \leq \mathbf{P} \mathbf{V}^{\oplus}(U ; I, X) \mathbf{p} \mathbf{v}^{\oplus}(f ; I) .
$$

Further $U^{\oplus} \diamond f^{\oplus}=U \diamond f^{\oplus}=U^{\oplus} \diamond f=U \diamond f$ a.e. on $I$,

$$
(U \diamond f)^{\oplus}(0)=U^{\oplus}(0) f^{\oplus}(0) .
$$


(c) If $U$ is Lipschitz continuous and $f \in L_{1}(I, X)$, then $U \star f$ is continuous with

$$
\|U \star f\|_{\infty} \leq \mathbf{P} \mathbf{V}_{\infty}(U ; I, X)\|f\|_{1} .
$$

If $U(t)$ and $V(t), t \in I$, are families of bounded linear operators which are of bounded strong pseudo-variation, then, for each $x, U \diamond(V(\cdot) x)$ is defined and a function of bounded pseudo-variation, but difficult to turn into a family of bounded linear operators $W(t) x$, because it is uniquely determined up to a set of Lebesgue measure 0 which depends on $x$. This situation can be remedied by setting

$$
(U \diamond V)(t) x=(U \diamond[V(\cdot) x])^{\oplus} .
$$

By Lemma 11 and Corollary 15 (b), $U \diamond V$ is a family of bounded linear operators that is of locally bounded strong pseudo-variation and strongly right-continuous.

Proposition 16. Let $f:[0, T) \rightarrow X$ be of bounded pseudo-variation and $U(t), V(t)$, $t \in[0, T)$, form families of bounded linear operators on $X$ that are of bounded strong pseudo-variation. Then

$$
(U \diamond V) \diamond f=U \diamond(V \diamond f) \quad \text { a.e. }
$$

We define recursively

$$
U^{\diamond 1}=U^{\oplus}, \quad U^{\diamond(n+1)}=U \diamond U^{\diamond n} .
$$

Corollary 17. Let $(U(t))_{t \in I}$ be a family of bounded linear operators that is of locally bounded strong variation. Then

$$
\mathbf{P V}^{\oplus}\left(U^{\diamond n} ; I, X\right) \leq\left[\mathbf{P} \mathbf{V}^{\oplus}(U ; I, X)\right]^{n} \quad \forall n \in \mathbf{N} .
$$

If $U(t)$ maps $X$ into a closed subspace $Z$, then

$$
\mathbf{P V}^{\oplus}\left(U^{\diamond(n+1)} ; I, X\right) \leq\left[\mathbf{P} \mathbf{V}^{\oplus}(U ; I, Z)\right]^{n} \mathbf{P V}^{\oplus}(U ; I, X) \quad \forall n \in \mathbf{N} .
$$

Application to integrated semigroups. If we apply our results to integrated semigroups we obtain the following generalization of Theorem 3. Notice that $S \diamond f=S \star f$ because $S^{\oplus}(0)=0$.

LEMMA 18. Let $S$ be an integrated semigroup which is of locally bounded strong pseudo-variation. Then $(S \diamond f)(t) \in \overline{D(A)}$ and $(S * f)(t) \in D(A)$ for all $f \in L_{1}(I, X)$ and almost all $t \geq 0$. Furthermore we have

$$
A(S * f)(t)=(S \diamond f)(t)-\int_{0}^{t} f(s) d s \quad \text { for a.a. } t \geq 0
$$

and

$$
(S * f)(t)=\int_{0}^{t}(S \diamond f)(s) d s \quad \forall t \in I .
$$

4. Cumulative outputs of bounded strong variation. Recall that $X, Y$ are Banach spaces and $A$ is a Hille-Yosida operator on $X$. The locally Lipschitz continuous integrated semigroup generated by $A$ is denoted by $(S(t))_{t \geq 0}$ and the $C_{0}$-semigroup on $\overline{D(A)}$ generated by $A_{0}$ is denoted by $(\dot{S}(t))_{t \geq 0}$. 
Assumption H4. Let $B$ be an $A$-bounded operator with values in $Y, T \in[0, \infty]$, such that for every $x \in X$,

$$
B \int_{0}^{t} S(s) x d s=\int_{0}^{t} V(s) x d s, \quad t \in[0, T),
$$

with a family $(V(t))_{t \geq 0}$ of bounded linear operators from $X$ to $Y$ that is of bounded strong pseudo-variation on $I=[0, T)$.

REMARK 1. Replacing $V$ by $V^{\oplus}$ we see that $B \int_{0}^{t} S(s) d s$ is right differentiable with derivative $V^{\oplus}(t) x$. So without restriction, we assume this from the very beginning, with $V(t)$ being strongly right continuous.

Lemma 19. Let Assumption $\mathrm{H}_{4}$ be satisfied. Then the following holds:

1. For all $x \in \overline{D(A)}$ and all $t \in I, B S(t) x=V(t) x$ and $V(\cdot) x$ is a.e. differentiable on $[0, T)$ with $\frac{d}{d t} V(\cdot) x \in L_{1}([0, T) ; X)$ and $V(t) x=\int_{0}^{t} \frac{d}{d s} V(s) x d s$.

For all $x \in D(A)$ and all $t \in I, V(t) x$ is right differentiable and

$$
B x+V(t) A x=V^{\prime}(t) x .
$$

For all $x \in D\left(A_{0}\right)$ and all $t \in I, V(t) x$ is continuously differentiable and $B \dot{S}(t) x=$ $V^{\prime}(t) x$.

2. For all $f \in L_{1}(I, X)$ and $t \in I$,

$$
(V * f)(t)=B(S * f)(t) .
$$

3. If $(x, f) \in E, E$ as in (13), then $V(\cdot) x+V * f$ is continuously differentiable on $[0, T]$ and

$$
B(\dot{S}(t) x+(S \diamond f)(t))=\frac{d}{d t}(V(t) x+(V * f)(t)) .
$$

In particular $V(\cdot) x \in W_{1, l o c}^{1}[0, T]$ and

$$
B(\dot{S}(t) x+(S \diamond f)(t))=V^{\prime}(t) x+(V * f)^{\prime}(t) .
$$

Proof. The first statement in 1 . is obvious because $A \int_{0}^{t} S(s) x d s$ is differentiable for $x \in$ $\overline{D(A)}$ with derivative $A S(t) x$. Similarly, for $x \in D\left(A_{0}\right), A S(t) x$ is differentiable with derivative $A \dot{S}(t) x=\dot{S}(t) A_{0} x$ and so is $B S(t) x=V(t) x$ with derivative $B \dot{S}(t) x$. For $x \in D(A)$, by part 1 and $(7)$,

$$
V(t) x=B t x+B \int_{0}^{t} S(s) A x d s=t B x+\int_{0}^{t} V(s) A x d s .
$$

We see that $V(t) x$ is right differentiable and

$$
V^{\prime}(t) x=B x+V(t) A x, \quad x \in D(A) .
$$

Returning to the case that $x \in D\left(A_{0}\right)$, we have that

$$
\int_{0}^{T}\left|V^{\prime}(t) x\right| d t=\mathbf{p} \mathbf{V}(V(\cdot) x ;[0, T)) \leq \mathbf{P V}(V ;[0, T), \overline{D(A)})\|x\| .
$$

If $x \in \overline{D(A)}=\overline{D\left(A_{0}\right)}$, we find a sequence $\left(x_{n}\right)$ in $D\left(A_{0}\right)$ such that $x_{n} \rightarrow x$ as $n \rightarrow \infty$. By the previous estimate, the functions $V^{\prime}(\cdot) x_{n}$ form a Cauchy sequence in the Banach 
space $L_{1}([0, T) ; X)$ and have a limit $v$ therein. Then

$$
\int_{0}^{t} v(s) d s=\lim _{n \rightarrow \infty} \int_{0}^{t} V^{\prime}(s) x_{n} d s=\lim _{n \rightarrow \infty} V(t) x_{n}=V(t) x .
$$

As for 2. we first recall that $A(S * f)(t)$ is continuous in $t$ (Theorem 3 ). Since $B$ is $A$-continuous, $B(S * f)(t)$ is continuous. Changing the order of integration shows that

$$
\int_{0}^{t}(S * f)(r) d r=\int_{0}^{t}\left(\int_{0}^{t-s} S(r) f(s) d r\right) d s .
$$

So

$$
\begin{aligned}
& \int_{0}^{t} B(S * f)(r) d r=B \int_{0}^{t}(S * f)(r) d r=\int_{0}^{t} B\left(\int_{0}^{t-s} S(r) f(s) d r\right) d s \\
& =\int_{0}^{t}\left(\int_{0}^{t-s} V(r) f(s) d r\right) d s=\int_{0}^{t}(V * f)(r) d r .
\end{aligned}
$$

Since $V * f$ is continuous, the assertion follows.

3. As $B$ is $A$-bounded and $A(\dot{S}(t) x+(S \diamond f)(t))$ is continuous,

$$
\begin{aligned}
& B(\dot{S}(t) x+(S \diamond f)(t)) \\
& =\lim _{h \rightarrow 0} \frac{1}{h} B(S(t+h) x-S(t) x+(S * f)(t+h)-(S * f)(t)) \\
& =\lim _{h \rightarrow 0} \frac{1}{h}(V(t+h) x-V(t) x+(V * f)(t+h)-(V * f)(t))
\end{aligned}
$$

uniformly for $t$ in bounded intervals. So $V(t) x+(V * f)(t)$ is continuously differentiable in $t$ with derivative $B(\dot{S}(t) x+(S \diamond f)(t))$.

It follows from Lemma $19(1)$ and the fact that $S(t)$ maps $X$ into $\overline{D(A)}$ that $V$ has the properties of a cumulative output defined below [12, 24, 32]. In some circumstances it is convenient to start from the operator family $V(t)$ rather than the operator $B$.

Definition 5. A family $(V(t))_{t \geq 0}$ of bounded operators from $X$ to $Y$ is a cumulative output for $(S(t))_{t \geq 0}$ if the following holds: $V(t)$ is strongly right continuous at any $t>0$, the right limit $V(0+)$ exists strongly, and

$$
V(t) S(r)=\int_{0}^{t+r} V(s) d s-\int_{0}^{t} V(s) d s-\int_{0}^{r} V(s) d s \quad \forall t, r \geq 0 .
$$

It follows from the uniform boundedness theorem and Lemma 4.14 in [32] that $V(t)$ is exponentially bounded.

REMARK 2. Since $S(\cdot) x$ is continuously differentiable for all $x \in \overline{D(A)}$ we obtain

$$
V(t) S^{\prime}(r) x=V(t+r) x-V(r) x, \quad t, r>0 .
$$

If $w(V)$ denotes the exponentially bound for $(V(t))_{t \geq 0}$, we define

$$
F(\lambda) x=\lambda \int_{0}^{\infty} e^{-\lambda t} V(t) x d t, \quad \lambda>w(V), x \in X .
$$

Since $V$ is a cumulative output for $S, F(\lambda)$ is a resolvent output of $A$, i.e.,

$$
F(\lambda)(\mu-A)^{-1}=\frac{1}{\mu-\lambda}(F(\lambda)-F(\mu)), \quad \lambda, \mu>w(V), \lambda \neq \mu .
$$


So we can define $\hat{B}=F(\lambda)(\lambda-A)$ and show that this definition is independent of $\lambda>w(V)$. A straightforward Laplace transform argument shows that Assumption $\mathrm{H} 4$ is satisfied with $\hat{B}$ replacing $B$.

5. Application to the semilinear Cauchy problem. We state the additional assumptions on $G$ and $A$.

Assumption H5. There exists a linear operator $B: D(A) \rightarrow Y$ into a Banach space $Y$, $T>0$ such that

$$
\|G(x)-G(y)\| \leq\|B(x-y)\| \quad \forall x, y \in D(A)
$$

and

$$
B \int_{0}^{t} S(r) x d r=\int_{0}^{t} V(r) x d r, \quad t \in[0, T), x \in X,
$$

with an operator family $(V(t))_{t \in[0, T)}$ from $X$ to $Y$ which is of bounded strong pseudovariation and $\mathbf{P} \mathbf{V}^{\oplus}(V ;[0, T), Z)<1$.

THEOREM 20. Under Assumption H5, there is a unique continuous semiflow $\Theta$ on $\overline{D(A)}$ such that the exponential Lipschitz condition (5) is satisfied and the orbits of $\Theta$ uniquely solve the Cauchy problem (3) in the $L_{1}$-Friedrichs sense $\left(L_{1}-F\right)$.

Proof. Let $T>0$. We show that, under Assumption H5, the assumptions of Theorem 8 are fulfilled with

$$
K_{1}=\mathbf{P V}(V ;[0, T), X), \quad K_{2}=\mathbf{P V}^{\oplus}(V ;[0, T), Z) .
$$

Let $(x, v),(y, w) \in E, E$ as in (13). By Lemma 19 and H5,

$$
\begin{aligned}
\| G(\dot{S}(t) x+(S \diamond v)(t))-G(\dot{S}(t) & y+(S \diamond w)(t)) \| \\
\leq & \|B(\dot{S}(t) x+(S \diamond v)(t))-B(\dot{S}(t) y+(S \diamond w)(t))\| \\
& =\left\|V^{\prime}(t)(x-y)+(V \diamond(v-w))(t)\right\|
\end{aligned}
$$

for a.a. $t \geq 0$. By Corollary 15 (a),

$$
\begin{aligned}
\int_{0}^{T} \| G(\dot{S}(t) x+(S \diamond & v)(t))-G(\dot{S}(t) y+(S \diamond w)(t)) \| d t \\
& \leq \mathbf{P V}(V ;[0, T), X)\|x-y\|+\mathbf{P V}^{\oplus}(V ;[0, T), Z)\|v-w\|
\end{aligned}
$$

This theorem will work well if we have enough information about the integrated semigroup $S$ generated by $A$. Aesthetically it may be more pleasing if all assumptions are in terms of $A$ (or its resolvent) and $G$.

Corollary 21. There exists a linear operator $B: D(A) \rightarrow Y$ into a Banach space $Y$ such that

$$
\|G(x)-G(y)\| \leq\|B(x-y)\| \quad \forall x, y \in D(A),
$$

and there exist $\mu>0$ and $\gamma \in(0,1)$ and $c>0$ such that

$$
\int_{0}^{\infty}\left\|B(\lambda+\mu-A)^{-(k+1)} x\right\| k \lambda^{k-1} d \lambda+\liminf _{\lambda \rightarrow \infty}\left\|B(\lambda-A)^{-1} x\right\| \leq \gamma\|x\|
$$

$$
\forall x \in Z, k \in \mathbf{N}
$$




$$
\int_{0}^{\infty}\left\|B(\lambda+\mu-A)^{-(k+1)} x\right\| k \lambda^{k-1} d \lambda \leq c\|x\| \quad \forall x \in \overline{D(A)}, k \in \mathbf{N} .
$$

Then there is a unique continuous semiflow $\Theta$ on $\overline{D(A)}$ such that the orbits of $\Theta$ uniquely solve the Cauchy problem (3) in the Friedrichs sense $\left(L_{1}-F\right)$ for all $x \in \overline{D(A)}$. Proof. By Theorem 6.8 and the subsequent remark in [34], there is a family of bounded operators $(V(t))_{t \geq 0}$ and some $w>0$ such that

$$
B(\lambda-A)^{-1} x=\lambda \int_{0}^{\infty} e^{-\lambda t} V(t) x d t \quad \forall \lambda>w, x \in X,
$$

and $V$ is of bounded strong (pseudo)-variation on every finite interval, $V(0)=0$. Moreover the strong right limit $V(0+)=V^{\oplus}(0)$ exists and

$$
V^{\oplus}(0) x=\lim _{\lambda \rightarrow \infty} B(\lambda-A)^{-1} x .
$$

For sufficiently large $\lambda>0$,

$$
\|G(x)-G(y)\| \leq\left\|B(\lambda-A)^{-1}\right\|\|(\lambda-A)(x-y)\| \quad \forall x, y \in D(A)
$$

and therefore $G$ is $A$-continuous. A straightforward Laplace transform argument shows that Assumption $\mathrm{H} 4$ is satisfied on every finite interval provided we find some $T>0$ with $\mathbf{P V}^{\oplus}(V ;[0, T), Z)<1$. To this end, let

$$
V^{\mu}(t)=e^{-\mu t} V(t)+\mu \int_{0}^{t} e^{-\mu s} V(s) d s .
$$

By Proposition 3.1 in [34],

$$
B(\lambda+\mu-A)^{-1}=\lambda \int_{0}^{\infty} e^{-\lambda t} V^{\mu}(t) d t .
$$

$V^{\mu}$ is of bounded pseudo-variation on $[0, \infty)$, and $\mathbf{P} \mathbf{V}^{\oplus}\left(V^{\mu} ;[0, \infty), Z\right) \leq \gamma$ as follows from [39] or [4] (see also [34], Theorem 4.2). It follows from Proposition 3.1 (c) in [34] that, for any $T>0$,

$$
\begin{aligned}
\limsup _{h \rightarrow 0} \frac{1}{h} \int_{0}^{T-h} e^{-\mu t}\|V(t+h) x-V(h) x\| d t+\left\|V^{\oplus}(0) x\right\| & \\
& \left.=\mathbf{p v}^{\oplus}\left(V^{\mu}(\cdot) x\right),[0, T)\right) \leq \gamma\|x\| \quad \forall x \in Z .
\end{aligned}
$$

So

$$
\mathbf{P V}^{\oplus}(V ;[0, T) ; Z)<\gamma e^{\mu T} .
$$

By choosing $T>0$ small enough we achieve that $\gamma e^{\mu T}<1$ and our assertion follows from Theorem 20.

6. Semilinear Miyadera-Voigt theorems. For densely defined $A, X=Z$ and $G=B$ we obtain a new proof of the well known Miyadera-Voigt perturbation theorem for $C_{0^{-}}$ semigroups [35] that makes no use of the Dyson-Phillips series and works for semi-linear perturbations of Miyadera-Voigt type. If the perturbation is of Kato type, the density of $D(A)$ can be dropped. 
TheOREM 22. Let $A$ be the generator of a $C_{0}$-semigroup $\dot{S}$ on a Banach space $X, Z \subseteq X$ be a closed subspace of $X$ and $Y$ be another Banach space. Let $D$ be a dense subspace of $X$ such that $D \cap Z$ is dense in $Z$. Let $G_{0}: D \rightarrow Z, B_{0}: D \rightarrow Y, B_{0}$ linear, such that

$$
\left\|G_{0}(x)-G_{0}(y)\right\| \leq\left\|B_{0}(x-y)\right\| \quad \forall x, y \in D,
$$

and the following conditions are satisfied:

1. $D \subseteq D(A), \quad \dot{S}(t) D \subseteq D$, and $B_{0} \dot{S}(\cdot) x$ continuous for all $x \in D$.

2. There are $\alpha, \lambda, K>0$ and $\gamma \in(0,1)$ such that

(a) $\int_{0}^{\alpha} e^{-\lambda t}\left\|B_{0} \dot{S}(t) x\right\| d t \leq K\|x\|$ for all $x \in D$.

(b) $\int_{0}^{\alpha} e^{-\lambda t}\left\|B_{0} \dot{S}(t) x\right\| d t \leq \gamma\|x\|$ for all $x \in D \cap Z$.

Then there is a unique A-continuous extension $G: D(A) \rightarrow Z$ of $G_{0}$ and a unique continuous semiflow $\Theta$ on $X$ such that the exponential Lipschitz condition (5) and the following are satisfied:

1. For all $x \in X, \Theta(\cdot, x)$ uniquely solves the Cauchy problem (3) in the Friedrichs sense $\left(L_{1}-F\right)$.

2. For all $x \in X$ and $t \geq 0$ we have

$$
\Theta(t, x)=\dot{S}(t) x+\lim _{h \rightarrow 0} \int_{0}^{t} \dot{S}(t-s) G\left(\frac{1}{h} \int_{s}^{s+h} \Theta(r, x) d r\right) d s .
$$

REMARK 3. In the proof we will show that $B_{0}$ is $\left.A\right|_{D}$ bounded and can be uniquely extended to an $A$-bounded linear operator $B: D(A) \rightarrow Y$, answering a question asked in [35], Remark 5.

This particular result even holds if condition 2. is replaced by the following weaker one: There are $\alpha, K>0$ such that

$$
\left\|\int_{0}^{t} B_{0} \dot{S}(r) x d r\right\| \leq K\|x\| \quad \forall x \in D, t \in[0, \alpha] .
$$

Proof. Define

$$
V(t) x=\int_{0}^{t} B_{0} \dot{S}(s) x d s, \quad t \geq 0, x \in D .
$$

Since $\dot{S}$ is a semigroup that leaves $D$ invariant,

$$
V(t) \dot{S}(r) x=V(t+r) x-V(r) x, \quad t, r \geq 0, x \in D .
$$

By our assumptions,

$$
\|V(t) x\| \leq e^{\lambda \alpha} K\|x\|, \quad x \in D, t \in[0, \alpha] .
$$

$V(t)$ can be uniquely extended to a bounded linear operator from $X$ to $Y$ for $t \in[0, \alpha]$, satisfying the same estimate. The functional relationship between $V$ and $\dot{S}$ shows inductively that $V(t)$ can be extended to a bounded linear operator from $X$ to $Y$ for $t \in[0, n \alpha]$, $n \in \mathbf{N}$,

$$
V(t) \dot{S}(r)=V(t+r)-V(r), \quad t, r \geq 0 .
$$

Cf. Remark 2. This functional relationship implies that $V$ is exponentially bounded,

$$
\|V(t)\| \leq \tilde{M} e^{\tilde{\omega} t}, \quad t \geq 0
$$


with appropriate constants $\tilde{M}, \tilde{\omega}$. See the proof of Lemma 4.14 in [32]. It also follows that $V(t) x \rightarrow 0, t \rightarrow 0$, first for $x \in D$, but then also for $x \in X$. By $(26), V(t)$ is strongly right continuous. Set

$$
F(\lambda)=\lambda \int_{0}^{\infty} e^{-\lambda t} V(t) d t, \quad \lambda>\tilde{\omega} .
$$

Taking Laplace transforms of the functional relation (26) between $V$ and $\dot{S}$ shows that $F(\cdot)$ is a resolvent output for $A$,

$$
F(\lambda)(\mu-A)^{-1}=\frac{1}{\mu-\lambda}(F(\lambda)-F(\mu)), \quad \mu, \lambda>\tilde{\omega}, \lambda \neq \mu .
$$

Thus the definition $B:=F(\lambda)(\lambda-A)$ is independent of $\lambda>\tilde{\omega}$ and provides an $A$-bounded operator $B$. Set

$$
S(t)=\int_{0}^{t} \dot{S}(r) d r
$$

Then

$$
B S(t) x=F(\lambda)(\lambda-A) S(t) x=F(\lambda)[\lambda S(t) x-\dot{S}(t) x+x]
$$

forms a strongly continuous exponentially bounded family of bounded linear operators. Taking Laplace transforms,

$$
\lambda \int_{0}^{\infty} e^{-\lambda t} B S(t) d t=F(\lambda)=\lambda \int_{0}^{\infty} e^{-\lambda t} V(t) d t .
$$

Since $B S(t)$ is strongly continuous and $V(t)$ strongly right continuous, the uniqueness properties of the Laplace transform imply that $V(t)=B S(t)$ for all $t \geq 0$ and the second formula in Assumption $\mathrm{H} 5$ follows. Further, for $x \in D$, both $V(t) x$ and $B S(t) x$ can continuously be differentiated yielding $B_{0} \dot{S}(t) x=B \dot{S}(t) x$ for all $t \geq 0$. Specializing to $t=0, B_{0} x=B x$, i.e., $B$ is an $A$-bounded extension of $B_{0}$.

Actually it follows from our assumptions that $D$ is a core for $A$, i.e., $D$ is dense in $D(A)$ with respect to the graph norm of $A$. See [13], Proposition 1.7, and [35], p. 168, where additional references can be found.

This allows to extend $G_{0}$ to a map $G$ from $D(A)$ to $Z$ as follows. Let $x \in D(A)$. Then there exists a sequence $\left(x_{n}\right)$ in $D$ such that $x_{n} \rightarrow x$ and $A x_{n} \rightarrow A x$ as $n \rightarrow \infty$. Since $B$ is an $A$-bounded extension of $B_{0}, B_{0} x_{n} \rightarrow B x$ as $n \rightarrow \infty$. The relation between $G_{0}$ and $B_{0}$ implies that $\left(G_{0}\left(x_{n}\right)\right)$ is a Cauchy sequence, and we can define

$$
G(x)=\lim _{n \rightarrow \infty} G_{0}\left(x_{n}\right) .
$$

This definition is independent of the choice of the sequence $\left(x_{n}\right)$ and

$$
\|G(x)-G(y)\| \leq\|B(x-y)\| \quad \forall x, y \in D(A) .
$$

Since $B$ is $A$-bounded, $G$ is an $A$-continuous extension of $G_{0}$, and as such uniquely determined by $G_{0}$ because $D$ is dense in $D(A)$ with respect to the graph norm of $A$.

By Lemma 19 (1), there exists a bounded linear map $\dot{V}: X \rightarrow L_{1}([0, \alpha], Y)$ such that $(\dot{V} x)(s)=B_{0} \dot{S}(s) x$ for $x \in D$ and

$$
V(t) x=\int_{0}^{t}(\dot{V} x)(s) d s
$$


and

$$
\begin{array}{ll}
\int_{0}^{\alpha} e^{-\lambda t}\|(\dot{V} x)(t)\| d t \leq K\|x\| & \forall x \in X \\
\int_{0}^{\alpha} e^{-\lambda t}\|(\dot{V} x)(t)\| d t \leq \gamma\|x\| & \forall x \in Z .
\end{array}
$$

Let $\tau \in(0, \alpha]$. Then

$$
\begin{aligned}
& \mathbf{p v}^{\oplus}(V(\cdot) x ;[0, \tau))=\int_{0}^{\tau}\|(\dot{V} x)(t)\| d t \leq K e^{\lambda \tau}\|x\| \quad \forall x \in X, \\
& \mathbf{p v}^{\oplus}(V(\cdot) x ;[0, \tau)) \leq \gamma e^{\lambda \tau}\|x\| \quad \forall x \in Z .
\end{aligned}
$$

Choosing $\tau>0$ small enough we can achieve that $\gamma e^{\lambda \tau}<1$. The existence and the properties of the semiflow $\Theta$ now follow from Theorem 20.

TheOREM 23. Let $A$ be a Hille-Yosida operator in a Banach space $X, A_{0}$ the part of $A$ in $\overline{D(A)}$ and $\dot{S}(\cdot)$ the $C_{0}$-semigroup generated by $A_{0}$.

Let $D$ be a dense subspace of $\overline{D(A)}$ and $Y$ another Banach space. Let $G_{0}: D \rightarrow X$, $B_{0}: D \rightarrow Y, B_{0}$ linear, such that

$$
\left\|G_{0}(x)-G_{0}(y)\right\| \leq\left\|B_{0}(x-y)\right\| \quad \forall x, y \in D,
$$

and the following conditions are satisfied:

1. $D \subseteq D\left(A_{0}\right), \quad \dot{S}(t) D \subseteq D$, and $B_{0} \dot{S}(\cdot) x$ continuous for all $x \in D$.

2. For every $\epsilon>0$ there exists $\delta>0$ such that

$$
\int_{0}^{\delta}\left\|B_{0} \dot{S}(t) x\right\| d t \leq \epsilon\|x\| \text { for all } x \in D
$$

Then there is a unique A-continuous extension $G: D(A) \rightarrow X$ of $G_{0}$ such that $G(x)=$ $\lim _{\lambda \rightarrow \infty} G\left(\lambda(\lambda-A)^{-1} x\right)$ for all $x \in D(A)$ and a unique continuous semiflow $\Theta$ on $X$ such that the exponential Lipschitz condition (5) and the following are satisfied:

1. For all $x \in X, \Theta(\cdot, x)$ uniquely solves the Cauchy problem (3) in the Friedrichs sense $\left(L_{1}-F\right)$.

2. For all $x \in X$ and $t \geq 0$ we have

$$
\Theta(t, x)=\dot{S}(t) x+\lim _{h \rightarrow 0} \int_{0}^{t} \dot{S}(t-s) G\left(\frac{1}{h} \int_{s}^{s+h} \Theta(r, x) d r\right) d s .
$$

Perturbations $\left(A+B_{0}\right)_{\mid D}$ of this kind are said to be of Kato type [37]. We mention that Hille-Yosida operators are preserved under Kato type perturbations ([34], Theorem $6.11)$.

Proof. The same proof as for Theorem 22 provides a strongly continuous family of bounded linear operators $V_{0}(t): \overline{D(A)} \rightarrow Y, V_{0}(0)=0$, with

$$
V_{0}(t) \dot{S}(r) x=V_{0}(t+r) x-V_{0}(r) x, \quad t, r \geq 0, x \in \overline{D(A)},
$$

and

$$
\mathbf{P V}^{\oplus}\left(V_{0} ;[0, \delta), \overline{D(A)}\right) \leq \epsilon,
$$

where $\delta$ has been chosen in dependence of $\epsilon$ as in 2. of the assumptions of this theorem. In particular $V_{0}(t) \rightarrow 0, t \rightarrow 0$, in operator norm. The functional relation with $\dot{S}$ implies 
that $V_{0}(t)$ is right continuous in operator norm. Integrating over $r$,

$$
\begin{gathered}
V_{0}(t) S(r) x=\int_{0}^{t+r} V_{0}(s) x d s-\int_{0}^{t} V_{0}(s) x d s-\int_{0}^{r} V_{0}(s) x d s, \\
t, r \geq 0, x \in \overline{D(A)} .
\end{gathered}
$$

Then $V_{0}$ can be extended to a cumulative output for $S$ on $X$ (i.e., (28) holds for $x \in X$ ) by

$$
V(t):=\lim _{h \searrow 0} V_{0}(t) \frac{1}{h} S(h)
$$

with convergence holding in operator norm (cf. Definition 5). See the proof of Theorem 6.11 in [34]. It is readily checked that $V$ is a cumulative output for $S$ on $X$. Further

$$
\mathbf{P V}^{\oplus}(V ;[0, \delta), X) \leq M \mathbf{P} \mathbf{V}^{\oplus}(V ;[0, \delta), \overline{D(A)}) \leq M \epsilon,
$$

with $M=\liminf _{h \searrow 0} \frac{1}{h}\|S(h)\|$. Choosing $\epsilon>0$ and $\delta>0$ small enough,

$$
\mathbf{P V}^{\oplus}(V ;[0, \delta), X)<1 \text {. }
$$

By Lemma 4.14 in [32], $V(\cdot)$ is exponentially bounded and, as in the proof of Theorem 22 , we define

$$
F(\lambda)=\lambda \int_{0}^{\infty} e^{-\lambda t} V(t) d t
$$

Then $F(\lambda) \rightarrow 0$ as $\lambda \rightarrow \infty$ in operator norm. By taking Laplace transforms of the cumulative output relation (28), for $V$ rather than $V_{0}, F(\cdot)$ is a resolvent output for $A$ and the definition $B=F(\lambda)(\lambda-A)$ is independent of $\lambda$. The uniqueness properties of the Laplace transform imply that

$$
B \int_{0}^{t} S(r) d r=\int_{0}^{t} V(r) d r .
$$

Let $\tilde{B}$ be another extension of $B_{0}$ to $D(A), \tilde{B}(\lambda-A)^{-1} \rightarrow 0, \lambda \rightarrow \infty$, in operator norm. Since $D$ is a core for $A_{0}, \tilde{B}$ and $B$ coincide on $D\left(A_{0}\right)$. Set $\tilde{F}(\lambda)=\tilde{B}(\lambda-A)^{-1}$. Then

$$
\tilde{B} x=\tilde{F}(\lambda)(\lambda-A) x=\lim _{\mu \rightarrow \infty} \mu \tilde{F}(\mu) x, \quad x \in D(A) .
$$

Similarly $B x=\lim _{\mu \rightarrow \infty} \mu F(\mu) x, x \in D(A)$. But $F(\lambda) x=B(\lambda-A)^{-1} x=\tilde{B}(\lambda-A)^{-1} x=$ $\tilde{F}(\lambda) x$ for $x \in D(A)$ because $(\lambda-A)^{-1}$ maps $D(A)$ into $D\left(A_{0}\right)$, so $\tilde{B}=B$.

By the definition of $B$,

$$
B x=\lim _{\lambda \rightarrow \infty} \lambda F(\lambda) x=\lim _{\lambda \rightarrow \infty} \lambda B(\lambda-A)^{-1} x, \quad x \in D(A) .
$$

As in the proof of Theorem $22, G_{0}$ has a unique $A_{0}$-continuous extension $\tilde{G}: D\left(A_{0}\right) \rightarrow X$ which satisfies

$$
\|\tilde{G}(x)-\tilde{G}(y)\| \leq\|B(x-y)\|, \quad x, y \in D\left(A_{0}\right) .
$$

It follows that, for $x \in D(A)$, the family $\left(\tilde{G}\left(\lambda(\lambda-A)^{-1} x\right)\right)$ is a Cauchy net as $\lambda \rightarrow \infty$. Hence the limit

$$
G(x)=\lim _{\lambda \rightarrow \infty} \tilde{G}\left(\lambda(\lambda-A)^{-1} x\right), \quad x \in D(A),
$$


exists and $G$ satisfies $\|G(x)-G(y)\| \leq\|B(x-y)\|$ for all $x, y \in D(A)$. Since $B$ is $A$-bounded, $G$ is $A$-continuous. Further $G$ is uniquely determined by $\tilde{G}$ and the property $G(x)=\lim _{\lambda \rightarrow \infty} G\left(\lambda(\lambda-A)^{-1} x\right)$ for all $x \in D(A)$.

We have checked all assumptions of Theorem 20 and our assertions follow.

7. Semilinear perturbations in Banach lattices. We consider a Banach lattice $X$ with positive convex cone $X_{+}$. Following [3, 1], we call a linear operator $A$ in $X$ resolvent positive, if $(a, \infty) \subseteq \rho(A)$ for some $a \in \mathbf{R}$ and $(\lambda-A)^{-1} \geq 0$ for all $\lambda>a$. Here $\rho(A)$ denotes the resolvent set of $A$. We refer to [26] for more details on ordered Banach spaces and positive operators.

We say that a linear operator $A$ in $X$ is absolutely resolvent majorized by a resolvent positive operator $\bar{A}$ if $(a, \infty) \in \rho(A) \cap \rho(\bar{A})$ for some $a \in \mathbf{R}$ with

$$
\left|(\lambda-A)^{-1} x\right| \leq(\lambda-\bar{A})^{-1}|x| \quad \text { for all } x \in X \text { and } \lambda>a .
$$

Given a family of bounded operators $(V(t))_{t \geq 0}$ of locally bounded strong variation we obtain iterative convolutions $V^{\diamond n}$ for $n \in \mathbf{N}$ by

$$
V^{\diamond(n+1)}:=\left(V^{\diamond n} \diamond V\right) \text {. }
$$

$V^{\diamond n}$ is again a family of bounded operators that is of locally bounded strong variation (see Corollary 17). We make the following assumption. Recall that an operator in a Banach lattice is called positive if it maps positive elements in its domain into $X_{+}$.

Assumption H7A. Let $X$ be a Banach lattice and $A$ be resolvent majorized by a resolvent positive Hille-Yosida operator $\bar{A}$. Let $G: D(A) \rightarrow Z$ map into a closed sublattice $Z$ of $X$ and assume that there is a linear positive operator $B: D(\bar{A}) \rightarrow Z$ such that

$$
|G(x)-G(y)| \leq B v \quad \text { whenever } \quad x, y \in D(A), v \in D(\bar{A}),|x-y| \leq v,
$$

and there exists some $w \in \mathbf{R}$ such that

$$
B(\lambda-\bar{A})^{-1} x=\lambda \int_{0}^{\infty} e^{-\lambda t} V(t) x d t \quad \forall \lambda>w, x \in X,
$$

where $V$ is a family of bounded strong pseudo-variation on some interval $[0, T+\epsilon)$ with $T, \epsilon>0$ and

$$
\mathbf{P V}^{\oplus}\left(V^{\diamond n} ;[0, T), Z\right)<1
$$

for some $n \in \mathbf{N}$.

REMARK 4. (a) Since $(\lambda-\bar{A})^{-1}$ and $B$ are positive we have

$$
|G(x)-G(y)| \leq B(\lambda-\bar{A})^{-1}|(\lambda-A)(x-y)|
$$

and therefore $G$ is $A$-continuous.

(b) Inductively one realizes that

$$
\left|(\lambda-A)^{-n} x\right| \leq(\lambda-\bar{A})^{-n}|x| \quad \forall x \in X, n \in \mathbf{N},
$$

which implies that $A$ is a Hille-Yosida operator as well.

We are going to prove a theorem similar to Theorem 20 and proceed as in the previous section by showing first that $\Psi_{x}$ is well defined and then that it admits a unique fixed point. Let $(\bar{S}(t))_{\geq 0}$ denote the integrated semigroup generated by $\bar{A}$. We start with 
Lemma 24. Let $T>0$. Under Assumptions $H^{r}$ a, $\Psi_{0}$ satisfies the inequalities (18) in Corollary 9 with

$$
K_{1}=\mathbf{P V}(V ;[0, T+), X), \quad K_{2}=\mathbf{P V}^{\oplus}(V ;[0, T+), Z),
$$

where

$$
\mathbf{P V}(V ;[0, T+), X):=\underset{h \downarrow 0}{\limsup } \mathbf{P V}(V ;[0, T+h), X)
$$

Further the extension $\Psi$ of $\Psi_{0}$ satisfies

$$
|\Psi(x, v)-\Psi(x, w)| \leq V \diamond|v-w| \quad \forall v, w \in L_{1}([0, T] ; Z) .
$$

Proof. We start by showing for $v \in L_{1}([0, T], X)$ that

$$
|(S \diamond v)(t)| \leq(\bar{S} \diamond|v|)(t)
$$

We have for locally Lipschitz continuous integrated semigroups (see [16] p.224)

$$
S(t+h) x-S(t) x=\lim _{k \rightarrow \infty} \int_{t}^{t+h}\left(\frac{k}{s}\right)\left(\frac{k}{s}-A\right)^{-(k+1)} x d s .
$$

Since $A$ is resolvent dominated by $\bar{A}$,

$$
\begin{aligned}
|(S(t+h)-S(t)) x| & \leq \lim _{k \rightarrow \infty} \int_{t}^{t+h}\left(\frac{k}{s}\right)\left|\left(\frac{k}{s}-A\right)^{-(k+1)} x\right| d s \\
\leq & \lim _{k \rightarrow \infty} \int_{t}^{t+h}\left(\frac{k}{s}\right)\left(\frac{k}{s}-\bar{A}\right)^{-(k+1)}|x| d s=(\bar{S}(t+h)-\bar{S}(t))|x|,
\end{aligned}
$$

and the inequality (29) follows a.e. from Theorem 14 and $S \diamond v=S \star v$. As a by-product, we see that $\{\bar{S}(t)\}$ is an increasing family. Since $S \diamond v$ and $\bar{S} \diamond|v|$ are continuous by Theorem 3, the inequality holds everywhere.

We apply Corollary 10. Let $\phi: \mathbf{R} \rightarrow[0, \infty)$ be infinitely often differentiable with support in $(0,1)$ and $\int_{0}^{1} \phi(s) d s=1$. Then, for $x, y \in \overline{D(A)}$,

$$
\begin{aligned}
\mid(\dot{S}(\cdot) x)_{j}^{\phi}(t)- & (\dot{S}(\cdot) y)_{j}^{\phi}(t)|=| \int_{0}^{\infty} \phi_{j}(s-t) \dot{S}(s)(x-y) d s \mid \\
& =\lim _{\lambda \rightarrow \infty}\left|\int_{0}^{\infty} \phi_{j}(s-t) \dot{S}(s) \lambda(\lambda-A)^{-1}(x-y) d s\right| \\
& =\lim _{\lambda \rightarrow \infty} \lim _{h \searrow 0}\left|\int_{0}^{\infty} \phi_{j}(s-t) \frac{1}{h}[S(s+h)-S(s)] \lambda(\lambda-A)^{-1}(x-y) d s\right| \\
& \leq \lim _{\lambda \rightarrow \infty} \lim _{h \searrow 0} \int_{0}^{\infty} \phi_{j}(s-t) \frac{1}{h}[\bar{S}(s+h)-\bar{S}(s)] \lambda(\lambda-\bar{A})^{-1}|x-y| d s \\
& =\lim _{\lambda \rightarrow \infty} \int_{0}^{\infty} \phi_{j}(s-t) \dot{\bar{S}}(s) \lambda(\lambda-\bar{A})^{-1}|x-y| d s .
\end{aligned}
$$


Since $\phi$ is zero on $(-\infty, 0]$,

$$
\begin{aligned}
& \left|(\dot{S}(\cdot) x)_{j}^{\phi}(t)-(\dot{S}(\cdot) y)_{j}^{\phi}(t)\right| \\
& \leq-\lim _{\lambda \rightarrow \infty} \int_{0}^{\infty} \phi_{j}^{\prime}(s-t) \bar{S}(s) \lambda(\lambda-\bar{A})^{-1}|x-y| d s \\
& =-\int_{0}^{\infty} \phi_{j}^{\prime}(s-t) \bar{S}(s)|x-y| d s=\int_{0}^{\infty} \phi_{j}^{\prime \prime}(s-t)\left(\int_{0}^{s} \bar{S}(r)|x-y| d r\right) d s \\
& \quad \in D(\bar{A}) .
\end{aligned}
$$

Let $v, w \in L_{1}([0, T], Z)$. We set $v(s)=w(s)=0$ for $s>T$. Let $t \in[0, T]$. Then

$$
\begin{aligned}
\left|(S \star v)_{j}^{\phi}(t)-(S \star w)_{j}^{\phi}(t)\right| \leq \int_{0}^{\infty} \phi_{j}(s-t)(\bar{S} \star|v-w|)(s) d s \\
=-\int_{0}^{\infty} \phi_{j}^{\prime}(s-t)(\bar{S} *|v-w|)(s) d s \in D(\bar{A}) .
\end{aligned}
$$

By Assumption H7a,

$$
\begin{aligned}
\left|G\left((\dot{S}(\cdot) x+S \star v)_{j}^{\phi}(t)\right)-G\left((\dot{S}(\cdot) y+S \star w)_{j}^{\phi}(t)\right)\right| \\
\leq B \int_{0}^{\infty} \phi_{j}^{\prime \prime}(s-t)\left(\int_{0}^{s} \bar{S}(r)|x-y| d r\right) d s \\
\quad-B \int_{0}^{\infty} \phi_{j}^{\prime}(s-t)(\bar{S} *|v-w|)(s) d s \\
=\int_{0}^{\infty} \phi_{j}^{\prime \prime}(s-t)\left(\int_{0}^{s} V(r)|x-y| d r\right) d s-\int_{0}^{\infty} \phi_{j}^{\prime}(s-t)(V *|v-w|)(s) d s \\
=\int_{0}^{\infty} \phi_{j}(s-t) V^{\oplus}(d s)|x-y|+\int_{0}^{\infty} \phi_{j}(s-t)(V \diamond|v-w|)(s) d s .
\end{aligned}
$$

Recall that $V^{\oplus}(t) x=V(t) x$ for a.a. $t$ and that $V^{\oplus}(\cdot)$ is of bounded strong variation which equals the strong pseudo-variation of $V(\cdot)$. So the Stieltjes integral in the last equation makes sense. Since the support of $\phi_{j}$ is contained in $(0,1 / j)$,

$$
\begin{aligned}
\int_{0}^{T}\left\|G\left((\dot{S}(\cdot) x+S \star v)_{j}^{\phi}(t)\right)-G\left((\dot{S}(\cdot) y+S \star w)_{j}^{\phi}(t)\right)\right\| d t \\
\leq \int_{0}^{T}\left\|\int_{0}^{\infty} \phi_{j}(s-t) V^{\oplus}(d s)|x-y|\right\| d t \\
\quad+\int_{0}^{T}\left(\int_{0}^{\infty} \phi_{j}(s-t)\|(V \diamond|v-w|)(s)\| d s\right) d t \\
\leq \mathbf{P V}\left(V ;\left[0, T+\frac{1}{j}\right), X\right)\|x-y\| \sup _{0 \leq s \leq T} \int_{0}^{\infty} \phi_{j}(s-t) d t \\
\quad+\int_{0}^{T+\frac{1}{j}}\left(\int_{0}^{\infty} \phi_{j}(s-t) d t\right)\|(V \diamond|v-w|)(s)\| d s
\end{aligned}
$$




$$
\begin{aligned}
\leq & \mathbf{P V}\left(V ;\left[0, T+\frac{1}{j}\right), X\right)\|x-y\| \\
& +\mathbf{P V}^{\oplus}\left(V ;\left[0, T+\frac{1}{j}\right), Z\right) \int_{0}^{T+\frac{1}{j}}\|v(s)-w(s)\| d s .
\end{aligned}
$$

In the last inequality we have used Corollary 15 (a). Recalling that we have set $v(t)=$ $0=w(t)$ for $t>T$, this implies the assumption in Corollary 10. Now

$$
\begin{aligned}
\mid \frac{1}{h} \int_{t}^{t+h}(S \star v)(s) d s & -\frac{1}{h} \int_{t}^{t+h}(S \star w)(s) d s \mid \\
& \leq \frac{1}{h} \int_{t}^{t+h}|(S \star(v-w))(s)| d s \leq \frac{1}{h} \int_{t}^{t+h}(\bar{S} \star|v-w|)(s) d s \\
& =\frac{1}{h}((\bar{S} *|v-w|)(t+h)-(\bar{S} *|v-w|)(t)) \in D(\bar{A}) .
\end{aligned}
$$

By Assumption H7a and Lemma 19 (2.),

$$
\begin{aligned}
\mid G\left(\frac{1}{h} \int_{t}^{t+h} \dot{S}(s) x d s+\frac{1}{h} \int_{t}^{t+h}\right. & (S \star v)(s) d s) \\
& -G\left(\frac{1}{h} \int_{t}^{t+h} \dot{S}(s) x d s+\frac{1}{h} \int_{t}^{t+h}(S \star w)(s) d s\right) \mid \\
\leq & B \frac{1}{h}((\bar{S} *|v-w|)(t+h)-(\bar{S} *|v-w|)(t)) \\
& =\frac{1}{h}((V *|v-w|)(t+h)-(V *|v-w|)(t)) .
\end{aligned}
$$

Then Corollaries 10 and 15 imply the second assertion.

We are now ready to prove a result similar to Theorem 20 for Banach lattices.

THEOREM 25. Under the assumption H7a there is a unique continuous semiflow $\Theta$ on $\overline{D(A)}$ satisfying (5) such that the orbits of $\Theta$ are the unique $F$-solutions in $L_{1}([0, T], X)$ of (10) for all $x \in X$ and $T>0$.

Proof. We want to apply Corollary 9. By Lemma 24, the first assumption holds and $\Psi_{0}$ has an extension $\Psi$. Therefore it only remains to show that there exists $n \in \mathbf{N}$ and $\varepsilon \in(0,1)$ such that

$$
\left\|\Psi_{x}^{n}(v)-\Psi_{x}^{n}(w)\right\|_{1} \leq \varepsilon\|v-w\|_{1} \quad \forall v, w \in L_{1}([0, T], Z), x \in \overline{D(A)} .
$$

By Lemma 24,

$$
\left|\Psi_{x}(v)-\Psi_{x}(w)\right| \leq V \diamond|v-w|
$$

Thus by induction

$$
\left|\Psi_{x}^{n}(v)-\Psi_{x}^{n}(w)\right| \leq V^{\diamond n} \diamond|v-w|
$$

By Corollary 15,

$$
\left\|\Psi_{x}^{n}(v)-\Psi_{x}^{n}(w)\right\|_{1} \leq \mathbf{P} \mathbf{V}^{\oplus}\left(V^{\diamond n} ;[0, T], X\right)\|v-w\|_{1} .
$$

and we have reached our goal with $\varepsilon=\mathbf{P V}^{\oplus}\left(V^{\diamond n} ;[0, T], X\right)$. 
We call a semiflow $\Theta$ on $\overline{D(A)}$ positive if $\Theta(t, \cdot)$ maps $\overline{D(A)} \cap X_{+}$into itself for all $t \geq 0$, where $X_{+}$denotes the positive cone of the Banach lattice $X$.

Corollary. Let $X$ be a Banach lattice and $A$ be a resolvent positive Hille-Yosida operator. Let $G: D(A) \rightarrow Z$ map into a closed subspace $Z$ of $X$ and be positive, i.e., $G$ maps $D(A) \cap X_{+}$into $X_{+}$.

Assume that there is a linear positive operator $B: D(A) \rightarrow Z$ such that

$$
|G(x)-G(y)| \leq B v \quad \text { whenever } x, y, v \in D(A),|x-y| \leq v,
$$

and there exists some $w \in \mathbf{R}$ such that

$$
B(\lambda-A)^{-1} x=\lambda \int_{0}^{\infty} e^{-\lambda t} V(t) x d t \quad \forall \lambda>w, x \in X .
$$

where $V$ is a family of bounded strong pseudo-variation on some interval $[0, T+\epsilon)$ with $T, \epsilon>0$ and

$$
\mathbf{P V}^{\oplus}\left(V^{\diamond n} ;[0, T), Z\right)<1 \quad \text { for some } n \in \mathbf{N} .
$$

Then there exists a unique continuous positive semiflow $\Theta$ on $\overline{D(A)}$ which satisfies (5) and whose orbits uniquely solve (3) in the Friedrichs sense $\left(L_{1}-F\right)$.

Proof. By Theorem 25 we only need to show the positivity of the semiflow. Since $G$ is positive and the integrated semigroup $S$ is increasing, $S \star v$ takes values in $X_{+}$if $v \in L_{1}\left([0, T], Z \cap X_{+}\right)$by Theorem 14 and the map

$$
\Psi(x, v)=\lim _{h \downarrow 0} G\left(\frac{1}{h}[S(t+h)-S(t)] x+\frac{1}{h} \int_{t}^{t+h}(S \star v)(s) d s\right)
$$

maps $\left(\overline{D(A)} \cap X_{+}\right) \times L_{1}\left([0, T], Z \cap X_{+}\right)$into itself. Hence, if $x \in \overline{D(A)} \cap X_{+}$, the fixed point $v_{x}$ is in $L_{1}\left([0, T], Z \cap X_{+}\right)$and

$$
\Theta(t, x)=\dot{S}(t) x+\left(S \star v_{x}\right)(t) \in X_{+}
$$

by Theorem 14 .

The Banach lattice $X$ is called an abstract $L$-space, if in addition the norm on $X$ is additive on the positive cone $X_{+}$(in particular $X$ may be a $L_{1}$-space or a space of measures).

Assumption H7B. Let $X$ be an abstract $L$-space, $A$ a linear operator in $X$, and

$$
G: D(A) \rightarrow X
$$

Suppose there are a resolvent positive Hille-Yosida operator $\bar{A}$ and a positive $\bar{A}$-bounded operator $B: D(\bar{A}) \rightarrow Z$ such that $A$ is absolutely resolvent majorized by $\bar{A}$,

$$
\text { spr } B(\mu-\bar{A})^{-1}<1 \quad \text { for some } \mu \in \mathbf{R} \cap \rho(\bar{A})
$$

and

$$
|G(x)-G(y)| \leq B w \quad \text { for all } x, y \in D(A), w \in D(\bar{A}) \text { with }|x-y| \leq w .
$$

THEOREM 26. The statement of Theorem 5.3 also holds if Assumption H7a is replaced by Assumption $\mathrm{H}^{\mathrm{r}} \mathrm{b}$. 
Proof. Since $\bar{A}$ is resolvent positive on $(w, \infty)$ for some $w>0$ and $B$ is positive, we have

$$
(-1)^{n} \frac{d^{n}}{d \lambda^{n}} B(\lambda-\bar{A})^{-1}=n ! B(\lambda-\bar{A})^{-(n+1)} \geq 0, \quad \lambda>w,
$$

i.e., $(B(\lambda-\bar{A}))_{\lambda>w}$ is completely monotonic. By the vector valued version of Bernstein's theorem (see [3], section 5, or [1], Section 2.7), we find an exponentially bounded increasing family $(V(t))_{t \geq 0}, V(0)=0$, such that

$$
B(\lambda-\bar{A})^{-1} x=\int_{0}^{\infty} e^{-\lambda t} d V(t) x=\lambda \int_{0}^{\infty} e^{-\lambda t} V(t) x d t, \quad \forall x \in X, \lambda>w .
$$

Since every increasing family of bounded operators is of bounded strong variation on every finite interval, we know from (23), and Theorem 14 that $\left(V^{\diamond n}(t)\right)_{t \geq 0}$ is an increasing family of bounded operators that is of bounded strong variation on every finite interval. Since $X$ is an abstract L-space and $V^{\diamond n}$ is increasing,

$$
\begin{aligned}
\int_{0}^{\epsilon-h} \| & V^{\diamond n}(s+h) x-V^{\diamond n}(s) x\left\|d s=\int_{0}^{\epsilon-h}\right\|\left|V^{\diamond n}(s+h) x-V^{\diamond n}(s) x\right| \| d s \\
= & \left\|\int_{0}^{\epsilon-h}\left|V^{\diamond n}(s+h) x-V^{\diamond n}(s) x\right| d s\right\| \leq\left\|\int_{0}^{\epsilon-h}\left(V^{\diamond n}(s+h)|x|-V^{\diamond n}(s)|x|\right) d s\right\| \\
= & \left\|\int_{\epsilon-h}^{\epsilon} V^{\diamond n}(s)|x| d s-\int_{0}^{h} V^{\diamond n}(s)|x| d s\right\| \leq h\left\|V^{\diamond n}(\epsilon)|x|-V^{\diamond n}(0)|x|\right\| .
\end{aligned}
$$

By Definition 3 and (20), for $n \geq 2$,

$$
\begin{aligned}
& \mathbf{p v}^{\oplus}\left(V^{\diamond n}(\cdot) x ;[0, \epsilon]\right)=\mathbf{p v}\left(V^{\diamond n}(\cdot) x ;[0, \epsilon]\right)+\left\|V^{\diamond n}(0) x\right\| \leq\left\|V^{\diamond n}(\epsilon)|x|\right\| \\
& \quad \leq e^{\lambda \epsilon}\left\|\int_{0}^{\epsilon} e^{-\lambda t} V^{\diamond n}(d t)|x|\right\| \leq e^{\lambda \epsilon}\left\|\left(B(\lambda-\bar{A})^{-1}\right)^{n}|x|\right\| \leq e^{\lambda \epsilon}\left\|\left(B(\lambda-\bar{A})^{-1}\right)^{n}\right\|\|x\| .
\end{aligned}
$$

By (21),

$$
\mathbf{P} \mathbf{V}^{\oplus}\left(V^{\diamond n} ;[0, \epsilon], X\right) \leq e^{\lambda \epsilon}\left\|\left(B(\lambda-\bar{A})^{-1}\right)^{n}\right\| .
$$

Choosing $\lambda$ and $n$ large and $\epsilon>0$ small enough, we have $\mathbf{P V}^{\oplus}\left(V^{\diamond n} ;[0, \epsilon], X\right)<1$ and our statement follows from Theorem 25.

8. Applications to age-structured population dynamics. In the sequel, we present applications to several age-structured population models. We treat the boundary condition at age 0 as in $[30,31,33,19]$. For alternative approaches see $[20,28]$ and the references cited there. The first model does not involve any additional structure, but has a very general birth law.

8.1. Age-structured population models without additional structure. The development of an age-structured population can be described as a partial differential equation for the age-density $u(t, \cdot)$ at time $t$,

$$
\begin{array}{cl}
\left(\frac{\partial}{\partial t}+\frac{\partial}{\partial a}\right) \frac{u(t, a)}{\mathcal{F}(a)}=0 ; & t>0,0<a<c, \\
u(t, 0)=f(u(t, \cdot)) ; & t>0, \\
u(0, a)=u_{0}(a) ; & 0 \leq a<c .
\end{array}
$$


The number $c \in(0, \infty]$ denotes the maximum age that an individual can reach. $\mathcal{F}(a)$ is the probability to survive till age $a$ and is a decreasing function of $a$ with $\mathcal{F}(0)=1$, $\mathcal{F}(a)>0$ for $a \in[0, c)$ and $\mathcal{F}(a)=0$ for $a>c$ with the last only making sense if $c<\infty$. $f(u(t, \cdot))$ is the population birth rate as a function of the population age density. We make the following assumption:

Assumption H8A. There exist a non-negative not necessarily finite Borel measure $m$ on $[0, c)$ such that $f: \mathcal{L}_{1}([0, c) ; m+\ell) \rightarrow[0, \infty), f(0)=0$, and

$$
|f(u)-f(v)| \leq \int_{[0, c)}|u(a)-v(a)| m(d a) .
$$

$\ell$ represents the Lebesgue measure and $\mathcal{L}_{1}([0, c) ; m+\ell)$ the space of real-valued Borel measurable functions $x$ such that

$$
\int_{[0, c)}|x(a)| m(d a)+\int_{[0, c)}|x(a)| d a<\infty .
$$

The assumption $f(0)=0$ means that the birth rate is 0 if the population size is 0 . As state space we choose the abstract $L$ space $X=\mathbf{R} \times L_{1}([0, c) ; \mathbf{R})$. However, the solutions $u$ of the abstract Cauchy problem in which we will recast (31) will take values in the subspace $\{0\} \times L_{1}([0, \infty) ; \mathbf{R})$ which can be identified with $L_{1}([0, \infty) ; \mathbf{R})$. We define the operator

$$
A(0, x)=\left(x(0),-\mathcal{F}\left(\frac{x}{\mathcal{F}}\right)^{\prime}\right), \quad x \in L_{1}([0, c)),
$$

with $D(A)$ being the set of functions $x \in L_{1}([0, c))$ such that $x / \mathcal{F}$ is absolutely continuous and $\mathcal{F}(x / \mathcal{F})^{\prime}$ is integrable on $[0, c)$. (Cf. [33], Section 5). We find that

$$
(\lambda-A)^{-1}(\xi, x)=(0, v), \quad \xi \in \mathbf{R}, x \in L_{1}[0, c),
$$

with

$$
v(a)=\xi e^{-\lambda a} \mathcal{F}(a)+\int_{0}^{a} e^{-\lambda(a-s)} \frac{\mathcal{F}(a)}{\mathcal{F}(s)} x(s) d s, \quad a \in[0, c) .
$$

We see that $(0, \infty)$ is contained in $\rho(A),\left\|(\lambda-A)^{-1}\right\| \leq 1 / \lambda$ for $\lambda>0$ and that $(\lambda-A)^{-1}$ maps the positive cone into itself, i.e., $A$ is resolvent-poitive. We make the following assumption:

Assumption H8B. There is some $w>0$ such that

$$
\int_{[s, c)} e^{-w(a-s)} \frac{\mathcal{F}(a)}{\mathcal{F}(s)} m(d a) \quad \text { is a bounded function of } s \in[0, c) .
$$

With this assumption, $D(A)$ is a subspace of $\mathcal{L}_{1}([0, c), m+\ell)$ and the following operator $B$ is defined on $D(A)$ :

$$
B(0, x)=\left(\int_{[0, c)} x(a) m(d a), 0\right), \quad x \in D(A) .
$$

Obviously $B$ is a positive operator from $D(A)$ to $X$. Further $G(x)=(f(x), 0)$ is an operator from $D(A)$ to $X$ satisfying the assumptions of Theorem 2, provided we can verify that the spectral radius of $F(\lambda)=B(\lambda-A)^{-1}$ is smaller than 1 . But $B$ maps 
$X$ into $Z=\mathbf{R} \times\{0\}$, so the spectral radius of $B(\lambda-A)^{-1}$ is smaller than the norm of $B(\lambda-A)^{-1}$ taken on this closed subspace, which is

$$
\left\|B(\lambda-A)^{-1}\right\|_{Z}=\int_{[0, c)} e^{-\lambda a} \mathcal{F}(a) m(d a) .
$$

This expression converges to $m(\{0\})$ for $\lambda \rightarrow \infty$. So it is sufficient to assume that

$$
m(\{0\})<1 .
$$

This is particularly satisfied if $m(\{0\})=0$, which means that an individual that has just been born does not reproduce.

TheOREM 27. Let the Assumptions H8a and H8b be satisfied and $m(\{0\})<1$. For each $u_{0} \in L_{1}([0, c), \mathbf{R})=L_{1}[0, c)$, there exists a unique Friedrichs solution $u$ which is defined for all times. More precisely $u(t, \cdot)$ is continuous in $t \geq 0$ as a function with values in $L_{1}[0, c), u(0, \cdot)=u_{0}$, and there exists a sequence of functions $u_{n}:[0, \infty) \times[0, c) \rightarrow \mathbf{R}$ with the following properties:

(a) For all $t \geq 0, u_{n}(t, \cdot) \in L_{1}[0, c)$ and

$$
\int_{0}^{c}\left|u_{n}(t, a)-u(t, a)\right| d a \rightarrow 0 \quad \text { locally uniformly in } t \geq 0
$$

(b) For all $t \geq 0, u_{n}(t, \cdot) / \mathcal{F}$ is absolutely continuous with $\mathcal{F} \frac{\partial}{\partial a} \frac{u_{n}(t, \cdot)}{\mathcal{F}} \in L_{1}[0, c), u_{n}(t, \cdot)$ is differentiable in $t$ as a function with values in $L_{1}[0, c)$, and

$$
\int_{0}^{T} \int_{0}^{c}\left|\frac{\partial}{\partial t} u_{n}(t, a)+\mathcal{F}(a) \frac{\partial}{\partial a} \frac{u_{n}(t, a)}{\mathcal{F}(a)}\right| d a d t \rightarrow 0, \quad n \rightarrow \infty
$$

where $\frac{\partial}{\partial t} u_{n}(t, a)$ stands for the derivative of $u_{n}(t, \cdot)$ with respect to $t$,

(c) $u_{n}(t, \cdot) \in L_{1}([0, c) ; m+\ell)$ and, for all $T>0$,

$$
\int_{0}^{T}\left|u_{n}(t, 0)-f\left(u_{n}(t, \cdot)\right)\right| d t \rightarrow 0, \quad n \rightarrow \infty .
$$

Moreover $\Theta\left(t, u_{0}\right)=u(t, \cdot)$ defines a semiflow on $L_{1}[0, c]$. If $f$ maps non-negative functions in $\mathcal{L}([0, c), m+\ell)$ onto non-negative functions, then $\Theta$ is a positive semiflow, i.e., if the initial datum $u_{0}$ is non-negative, so is the solution $u$.

8.2. An age-structured population model with an additional structure. Let $E$ be a Banach space that represents the distribution of a population with respect to a structure different from age, e.g., induced by space or body size. Let $u(t, a)$ denote the structural distribution (with respect to this structure) of the individuals with age $a$ at time $t$. We consider the following model:

$$
\begin{cases}\left(\partial_{t}+\partial_{a}\right) u(t, a)=A u(t, a)+M(a) u(t, a), & t>0,0<a<c \\ u(t, 0)=\int_{0}^{c}[F(u(t, \cdot))](a) d a, & t>0, \\ u(0, a)=u_{0}(a), & 0 \leq a<c .\end{cases}
$$

The number $c \in(0, \infty)$ denotes the maximum age an individual can reach. The closed linear operator $A$ and the bounded linear operators $M(a)$ describe how individuals (with age $a$ ) change with respect to the other structure and also to what extent they die. 
The boundary condition describes the birth of individuals. The operators $F(f)(a)$ represents the birth rate of individuals with age $a$ if $f:[0, c) \rightarrow E$ is the age-density of the population.

We assume that $A$ is the infinitesimal generator of a $C_{0}$-semigroup $\{U(t) ; t \geq 0\}$ on $E$. Then the set

$$
D=\left\{f \in C^{1}([0, c] ; E) ; f(0)=0=f^{\prime}(0), f(t) \in D(A) \quad \forall t \in[0, c], \text { Af continuous }\right\}
$$

is dense in $L_{1}([0, c), E)$.

For more general assumptions that allow the operator $A$ to depend on $a$ see [25]. We want to apply the semilinear Miyadera-Voigt theory developed in Section 6.

Assumption 28. Let the operators $F: D \rightarrow E$ and $M(a): E \rightarrow E, a \in[0, c)$, have the following properties:

(a) There exist a Banach space $\tilde{E}$ and an $A$-bounded linear operator $C: D(A) \rightarrow \tilde{E}$ such that

$$
\|[F(f)](a)-[F(g)](a)\| \leq\|C(f(a)-g(a))\| \quad \forall f, g \in D, a \in[0, c),
$$

and, for each $\epsilon>0$, there exists some $\delta \in(0, c)$ with

$$
\int_{0}^{\delta}\|C U(t) x\| d t \leq \epsilon\|x\| \quad \forall x \in D(A) .
$$

(b) $\{M(a) ; a \in[0, c)\}$ is a strongly Borel measurable family of bounded linear operators such that, for every $x \in E,\|x\| \leq 1$,

$$
\underset{0 \leq a<c}{\operatorname{ess} \sup }\|M(a) x\|<\infty .
$$

Following the approach in $[31,19]$ and $\left[33\right.$, Sec. 5], we let let $X_{\circ}=L_{1}([0, c), E)$. The evolutionary semigroup on $X_{\circ}$ associated with $U$ is defined by

$$
\left(T_{0}(t) f\right)(a)=\left\{\begin{array}{cc}
U(t) f(a-t), & a \geq t \\
0, & a<t
\end{array}\right\}, \quad f \in X_{\circ}, 0 \leq a<c .
$$

Cf. [7, 25], e.g., and the references therein. It is readily checked that $T_{0}(\cdot)$ is a $C_{0^{-}}$ semigroup on $X_{\circ}$ and $\left\|T_{0}(t)\right\| \rightarrow 0$ as $t \rightarrow \infty$. So there exist $\omega>0$ and $\hat{M} \geq 1$ such that $\left\|T_{0}(t)\right\| \leq \hat{M} e^{-\omega t}$. If $\mathcal{A}_{0}$ denotes the infinitesimal generator of $T_{0}, D \subseteq D\left(\mathcal{A}_{0}\right)$ and

$$
\left[\mathcal{A}_{0}(f)\right](a)=A f(a)-f^{\prime}(a), \quad f \in D, a \in[0, c) .
$$

Moreover $T_{0}(t) D \subseteq D$ for all $f \in D$. Let $X=E \times X_{\circ}$. It follows from the considerations in [33, Sec. 5], that there exists a Hille-Yosida operator $\mathcal{A}$ such that

$$
\begin{aligned}
& (\lambda-\mathcal{A})^{-1}(\eta, f)=(0, g), \\
& g(a)=e^{-\lambda a} U(a) \eta+\int_{0}^{a} e^{-\lambda s} U(s) f(a-s) d s .
\end{aligned}
$$

$\mathcal{A}$ generates an integrated semigroup $S(\cdot)$ on $X$ such that $\dot{S}(t)(0, v)=\left(0, T_{0}(t) v\right)$ and

$$
S(t)=\lim _{\lambda \rightarrow \infty} \int_{0}^{t} \lambda \dot{S}(r)(\lambda-\mathcal{A})^{-1} d r
$$


from which we deduce that

$$
\|S(t)-S(r)\| \leq \tilde{M} \int_{r}^{t} e^{-\omega s} d s
$$

and

$$
\left\|(\lambda-\mathcal{A})^{-n}\right\| \leq \tilde{M}(\lambda+\omega)^{-n} \quad \forall \lambda>-\omega, n \in \mathbb{N} .
$$

In terms of the original semigroup, the integrated semigroup is given by

$$
\begin{aligned}
& S(t)(\eta, f)=(0, g), \quad \eta \in E, f \in X_{\circ}, \\
& g(a)=H(t-a) U(a) \eta+\int_{0}^{t}\left[T_{0}(s) f\right](a) d s .
\end{aligned}
$$

Here $H$ denotes the Heaviside function, $H(t)=1$ for $t \geq 0, H(t)=0$ for $t<0$. The generator $\mathcal{A}$ of the integrated semigroup $S$ is related to the infinitesimal generator $A$ of $U$ by

$$
\mathcal{A}(0, f)=\left(-f(0), A f(\cdot)-f^{\prime}\right),
$$

for $f \in C^{1}([0, c], E)$ with $f(a) \in D(A)$ for all $a \in[0, c)$. Obviously $\overline{D(\mathcal{A})}=\{0\} \times X_{\circ}$ which can be identified with $X_{\circ}$. In order to make the connection to Theorem 23, we identify $D$ and $\{0\} \times D$ and define operators $B_{0}: D \rightarrow Y, Y=L_{1}([0, c), \tilde{E}) \times L_{1}([0, c), E)$, and $G_{0}: D \rightarrow X$ by

$$
\left.\begin{array}{c}
B_{0}(0, f)=(C f(\cdot), M(\cdot) f(\cdot)) \\
G_{0}(0, f)=\left(\int_{0}^{c}[F(f)](a) d a, M(\cdot) f(\cdot)\right)
\end{array}\right\} f \in D .
$$

The assumptions of Theorem 23 are readily checked, and there exists a semiflow on $L_{1}([0, c), E)$ which solves the age-structured problem 32 in an appropriate generalized sense.

8.3. Age-structured population models with diffusion. We make the additional structure in the preceding example explicit and assume that the individuals are distributed over a bounded open habitat $\Omega \subseteq \mathbf{R}^{N}$. If, at time $t, u(t, a, x)$ is the density of individuals at age $a$ and location $x$, then the model reads

$$
\begin{aligned}
& \left(\frac{\partial}{\partial t}+\frac{\partial}{\partial a}\right) u(t, a, x)=\gamma(x) \Delta_{x} u(t, a, x) \\
& -\mu(a, x) u(t, a, x) ; \quad x \in \Omega, \\
& u(t, a, x)=0 \\
& u(t, 0, x)=\int_{0}^{\infty} \beta_{0}(a, x) u(t, a, x) d a \\
& +\sum_{j=1}^{n} \beta_{j}(a, x)\left|\frac{\partial}{\partial x_{j}} u(t, a, x)\right| d a ; x \in \Omega, \\
& u(0, a, x)=u_{0}(a, x) ; \\
& x \in \partial \Omega \text {, } \\
& x \in \Omega \text {. }
\end{aligned}
$$

The number $c \in(0, \infty)$ denotes the maximum age that an individual can reach. $\mu(a, x)$ is the per capita mortality rate at age $a$ at location $x$. The individuals move randomly with a diffusion rate $\gamma(x)$ which only depends on location. $\Delta_{x}$ denotes the Laplace operator with respect to $x$. We assume that the boundary of the habitat $\Omega$ is deadly expressed 
by Dirichlet boundary conditions. The birth rate at location $x$ is assumed to depend not only on the density of individuals at $x$ but also on the magnitude of the flux through $x$. This dependence is expressed by the rates $\beta_{j}$.

We assume, for simplicity, that the boundary of $\Omega$ is of class $C^{3}$ and that $\mu$ is continuously differentiable on $[0, c) \times \Omega$ with bounded partial derivatives. Further we assume that $\gamma$ has bounded continuous partial derivatives up to order three. Moreover $\inf \{\gamma(x) ; x \in \Omega\}>0$. For sharper assumptions see the literature mentioned below. The functions $\beta_{j}$ are assumed to be non-negative, Borel measurable, and essentially bounded on $[0, c) \times \Omega$.

In order to connect the model (33) to the model (32), we let $E=L_{2}(\Omega), D(A)=$ $H^{2}(\Omega) \cap H_{0}^{1}(\Omega)$, and

$$
[A \phi](x)=\gamma(x) \Delta \phi(x), \quad[M(a) \phi](x)=-\mu(a, x) \phi(x) .
$$

Here $H^{k}(\Omega)$ is the Banach space of functions in $L_{2}(\Omega)$ whose generalized partial derivatives are also contained in $L_{2}(\Omega)$, up to order $k$; the norm on $H^{k}(\Omega)$ combines the $L_{2}$-norms of the function and its generalized derivatives up to order $k$ via the Euclidean norm. $H_{0}^{k}(\Omega)$ is the closure of $C_{0}^{\infty}(\Omega)$ in $H^{k}(\Omega)$. Further we set

$$
([F(\phi)](t))(x)=\beta_{0}(t, x)|\phi(x)|+\sum_{j=1}^{N} \beta_{j}(t, x)\left|\frac{\partial}{\partial x_{j}} \phi(x)\right|, \quad \phi \in H_{0}^{1}(\Omega) .
$$

Under our assumptions on $\gamma$ and $\mu, A$ generates a $C_{0}$-semigroup $\{U(t) ; t \geq 0\}$ with $U(t) \phi \in D(A)$ for all $t>0, \phi \in E$, which is given by a Green's function,

$$
[U(t) \phi](x)=\int_{\Omega} \Gamma(t, x, y) \phi(y) d y .
$$

See $[18$, Thm. 16.2, 16.3] and [14, Sec. VI.2.1]. In order to check the Assumptions 28, we set $\tilde{E}=E^{N+1}$, with the norm $\left\|\left(\phi_{0}, \ldots, \phi_{n}\right)\right\|=\sum_{j=0}^{N}\left\|\phi_{j}\right\|$. Then, for $\phi, \psi \in H_{0}^{1}(\Omega)$,

$$
\begin{aligned}
\|F(t) \phi-F(t) \psi\| & =\left\|\beta_{0}(t, \cdot)|\phi|+\sum_{j=1}^{N} \beta_{j}(t, \cdot)\left|\frac{\partial}{\partial x_{j}} \phi\right|-\beta_{0}(t, \cdot)|\psi|-\sum_{j=1}^{N} \beta_{j}(t, \cdot)\left|\frac{\partial}{\partial x_{j}} \psi\right|\right\| \\
& \leq\left\|\beta_{0}(t, \cdot)|\phi-\psi|+\sum_{j=1}^{N} \beta_{j}(t, \cdot)\left|\frac{\partial}{\partial x_{j}}(\phi-\psi)\right|\right\| .
\end{aligned}
$$

Since the functions $\beta_{j}$ are bounded, there exists a constant $\kappa$ such that

$$
\begin{aligned}
\|F(t) \phi-F(t) \psi\| & \leq\left\|\kappa|\phi-\psi|+\sum_{j=1}^{N} \kappa\left|\frac{\partial}{\partial x_{j}}(\phi-\psi)\right|\right\| \\
& \leq\|\kappa \phi-\kappa \psi\|+\sum_{j=1}^{N}\left\|\kappa \frac{\partial}{\partial x_{j}} \phi-\kappa \frac{\partial}{\partial x_{j}} \psi\right\|=\sqrt{N+1}\|C \phi-C \psi\|,
\end{aligned}
$$

where $C: H_{0}^{1}(\Omega) \rightarrow \tilde{E}, \tilde{E}=E^{N+1}$ is the linear operator

$$
C \phi=\kappa(\phi, \nabla \phi), \quad \phi \in H_{0}^{1}(\Omega) .
$$


Let $\phi \in E, t>0$. Then

$$
\begin{aligned}
C U(t) \phi & =\kappa\left(\phi_{0}, \ldots, \phi_{n}\right), \\
\phi_{0}(x) & =\int_{\Omega} \Gamma(t, x, y) \phi(y) d y, \\
\phi_{j}(x) & =\int_{\Omega} \frac{\partial}{\partial x_{j}} \Gamma(t, x, y) \phi(y) d y .
\end{aligned}
$$

The Green's function satisfies the following estimates [18, Thm. 16.3]:

$$
\begin{aligned}
0 \leq \Gamma(t, x, y) & \leq \xi_{0}(t,|x-y|), \\
\left|\frac{\partial}{\partial x_{j}} \Gamma(t, x, y)\right| & \leq \xi_{1}(t,|x-y|), \\
\xi_{i}(t, r) & =c_{1} t^{-\frac{N+i}{2}} \exp \left(-c r^{2} t^{-1}\right), \quad i=0,1 .
\end{aligned}
$$

With these estimates,

$$
\begin{aligned}
\left\|\phi_{j}\right\| & \leq \int_{\mathbf{R}^{N}} \xi_{1}(t,\|x\|) d x\|\phi\|=c_{N} \int_{0}^{\infty} \xi_{1}(t, r) r^{N-1} d r\|\phi\| \\
& =c_{N} c_{1} t^{-\frac{i}{2}} \int_{0}^{\infty} e^{-c r^{2}} d r\|\phi\|=\tilde{c}_{N} t^{-\frac{i}{2}}\|\phi\|,
\end{aligned}
$$

where $i=0$ for $j=0$ and $i=1$ for $j=1, \ldots, N$ and $c_{N}, \tilde{c}_{N}$ are appropriate constants. If $0 \leq t \leq 1$, with a constant $\hat{c}_{N}$,

$$
\|C U(t) \phi\| \leq \hat{c}_{N} t^{-\frac{1}{2}}\|\phi\|
$$

If $\delta \in(0,1]$, for all $\phi \in E$,

$$
\int_{0}^{\delta}\|C U(t) \phi\| d t \leq \hat{c}_{N} \int_{0}^{\delta} t^{-\frac{1}{2}}\|\phi\| d t \leq 2 \hat{c}_{N} \delta^{1 / 2}\|\phi\| .
$$

Acknowledgements. The authors thank Jürgen Voigt and Pierre Magal for useful comments.

\section{References}

[1] W. Arendt, C. J. K. Batty, M. Hieber, and F. Neubrander, Vector-valued Laplace Transforms and Cauchy Problems, Birkhäuser, Basel, 2001.

[2] W. Arendt, Vector valued Laplace transforms and Cauchy problems, Israel J. Math. 59 (1987), 327-352.

[3] W. Arendt, Resolvent positive operators, Proc. London Math. Soc. 54 (1987), 321-359.

[4] W. Arendt, Vector-valued versions of some representation theorems in real analysis, in: Functional Analysis, K. D. Bierstedt et al. (eds.), Marcel Dekker, New York, 1994, 33-50.

[5] Ph. Bénilan, M. G. Crandall, and A. Pazy, Bonnes solutions d'un problème d'évolution semi-linéaire, C. R. Acad. Sci. Paris Série I 306 (1988), 527-530.

[6] Ph. Bénilan and P. Wittbold, Nonlinear evolution equations in Banach spaces: basic results and open problems, in: Functional Analysis, K. D. Bierstedt et al. (eds.), Marcel Dekker, New York, 1994, 1-32.

[7] C. Chicone, Y. Latushkin, Evolution Semigroups in Dynamical Systems and Differential Equations, AMS, Providence, 1999. 
[8] S-N. Chow and J. K. Hale, Dynamics of Infinite Dimensional Systems, Springer, Berlin, 1987.

[9] M. G. Crandall and T. Liggett, Generation of semigroups on nonlinear transformations on general Banach spaces, Amer. J. Math. 93 (1971), 265-298.

[10] G. da Prato and E. Sinestrari, Differential operators with nondense domain, Ann. Scuola Norm. Sup. Pisa 14 (1987), 285-344.

[11] O. Diekmann, S. A. van Gils, S. M. Verduyn Lunel, and H.-O. Walther, Delay Equations. Functional, Complex, and Nonlinear Analysis, Springer, New York, 1995.

[12] O. Diekmann, M. Gyllenberg, and H. R. Thieme, Perturbing semigroups by solving Stieltjes renewal equations, Differential Integral Equations 6 (1993), 155-181

[13] K.-J. Engel and R. Nagel, One-Parameter Semigroups for Linear Evolution Equations, Springer, Berlin, 2000.

[14] M. G. Garroni and J. L. Menaldi, Green Functions for Second Order Parabolic Integrodifferential Problems, Longman Scientific \& Technical, Harlow, 1992.

[15] J. K. Hale, Asymptotic Behaviour of Dissipative Systems, AMS, Providence, 1988.

[16] E. Hille and R. Phillips, Functional Analysis and Semigroups, AMS, Providence, 1957.

[17] H. Kellermann and M. Hieber, Integrated semigroups, J. Funct. Anal. 84 (1989), 160-180.

[18] O. A. Ladyženskaja, V. A. Solonnikov, and N. N. Ural'ceva, Linear and Quasi-linear Equations of Parabolic Type, AMS, Providence, 1968.

[19] P. Magal, Compact attractors for time periodic age-structured population models, Electronic J. Differential Equations 2001 (2001), No. 65, 1-35.

[20] T. Matsumoto and S. Oharu, Semilinear evolution equations with nonlinear constraints and applications, J. Evol. Equ. 2 (2002), 197-222

[21] T. Matsumoto, S. Oharu, and H. R. Thieme, Nonlinear perturbations of a class of integrated semigroups, Hiroshima Math. J. 26 (1996), 433-473.

[22] I. Miyadera, On perturbation theory for semi-groups of operators, Tohoku Math. J. 18 (1966), 299-310.

[23] F. Neubrander, Integrated semigroups and their applications to the abstract Cauchy problem, Pac. J. Math. 135 (1988), 111-155.

[24] S. Piskarev and S.-Y. Shaw, Multiplicative perturbations of $C_{0}$-semigroups and some applications to step responses and cumulative outputs, J. Funct. Analysis 128 (1995), 315-340.

[25] F. Räbiger, R. Schnaubelt, A. Rhandi and J. Voigt, Non-autonomous Miyadera perturbations, Differential Integral Equations 13 (2000), 341-368.

[26] H. H. Schaefer, Banach Lattices and Positive Operators, Springer, Berlin, 1974.

[27] G. R. Sell and Y. You, Dynamics of Evolutionary Systems, Springer, New York, 2002.

[28] C. N. Shanthidevi, T. Matsumoto, and S. Oharu, Semilinear evolution equations with nonlinear constraints in age structured proliferating cell populations, preprint.

[29] H. R. Thieme, Integrated semigroups and integral solutions to abstract Cauchy problems, J. Math. Anal. Appl. 152 (1990), 416-447.

[30] H. R. Thieme, Semiflows generated by the Lipschitz perturbations of non-densely defined operators, Differential Integral Equations 3 (1990), 1035-1066.

[31] H. R. Thieme, Analysis of age-structured population models with an additional structure, in: Mathematical Population Dynamics, O. Arino (eds.), Marcel Dekker, New York, 1991, $115-126$.

[32] H. R. Thieme, Positive perturbations of dual and integrated semigroups, Adv. Math. Sci. Appl. 2 (1996), 445-507. 
[33] H. R. Thieme, Positive perturbations of operator semigroups: Growth bounds, essential compactness and asynchronous exponential growth, Discrete and Continuous Dynamical Systems 4 (1998), 735-764.

[34] H. R. Thieme and H. Vosseler, A Stieltjes type convolution for integrated semigroups of strong bounded variation and $L_{p}$-solutions to the abstract Cauchy problem, Differential Integral Equations 15 (2002), 1171-1218.

[35] J. Voigt, On the perturbation theory for strongly continuous semigroups, Math. Ann. 229 (1977), 163-171.

[36] J. Voigt, On resolvent positive operators and positive $C_{0}$-semigroups on $A L$-spaces, Semigroup Forum 38 (1989), 263-266.

[37] J. Voigt, Absorption semigroups, Feller property, and Kato class, in: Partial Differential Operators and Mathematical Physics, M. Demuth and B.-W. Schulze (eds.), Birkhäuser, Basel, 1995, 389-396.

[38] G. F. Webb, Continuous nonlinear perturbations of linear accretive operators in Banach spaces, J. Funct. Anal. 10 (1972), 191-203.

[39] L. Weis, Inversion of the vector valued Laplace transform in $L_{p}(X)$-spaces, in: Differential Equations in Banach Spaces, G. Dore et al. (eds.), Marcel Dekker, New York, 1993, 235253. 